\title{
Global games with strategic complements and substitutes
}

\author{
By
}

\author{
Eric J. Hoffmann* and Tarun Sabarwal ${ }^{\dagger}$
}

\begin{abstract}
We extend the global games method to the class of finite player, finite action games that includes games with strategic complements, games with strategic substitutes, and arbitrary combinations of the two. Our result is based on common order properties present in both strategic complements and substitutes, the notion of $p$-dominance, and the use of dominance solvability as the solution concept. In addition to being closer to the original arguments in Carlsson and van Damme (1993), our approach requires fewer additional assumptions. In particular, we require only one dominance region, and no assumptions on state monotonicity, or aggregative structure, or overlapping dominance regions. As expected, the $p$-dominance condition becomes more restrictive as the number of players increases. In cases where the probabilistic burden in belief formation may be reduced, the $p$-dominance condition may be relaxed as well. We present examples that are not covered by existing results.
\end{abstract}

JEL Numbers: C70, C72

Keywords: Global games, strategic complements, strategic substitutes, monotone games, equilibrium selection

First Draft: November 2012

This Version: August 13, 2019

${ }^{*}$ Department of Accounting, Economics, and Finance, West Texas A \& M University, Canyon TX 79016. Email: ehoffmann@wtamu.edu

${ }^{\dagger}$ Department of Economics, University of Kansas, Lawrence KS 66045. Email: sabarwal@ku.edu. 


\section{Introduction}

The global games method proposed by Carlsson and van Damme (1993), denoted CvD, serves as an equilibrium selection device for complete information games by embedding them into a class of Bayesian games based on noisy signals of payoff relevant states. CvD study 2-player, 2-action coordination games and show that as signals become more precise, a unique serially undominated prediction emerges in the associated Bayesian game. This

yields a unique Nash equilibrium in the original game. A key feature of this method is a contagion argument: Strictly dominant actions in particular states can infect behavior in states further away in which actions are no longer strictly dominant. Another key feature is that the selected equilibrium is risk dominant.

The global games method has since been extended by Frankel, Morris, and Pauzner (2003), denoted FMP, to multiple-player, multiple-action games of strategic complements (GSC). In such games a higher action from opponents induces a player to best respond with a higher action as well. Their framework encompasses many useful applications and increases the scope of global games.

Less is known about the applicability of global games more generally. Part of the reason for this is the complexity of the analytical framework. It requires a delicate balance among many moving components, including characteristics of player payoffs, role of dominance regions, structure of uncertainty and beliefs about signals of other players, properties of strategic interaction among players and their effect on serially undominated strategies, and then investigating limiting behavior of these combined interactions as noise goes to zero.

Indeed, as shown by Morris and Shin (2005), for games with strategic substitutes (GSS), in which a higher action from opponents induces a player to best respond with a lower action, the global games method can be very complex to apply, and they give an example of a GSS where this method fails to produce a unique outcome consistent with serially undominated strategies. More recently, Harrison and Jara-Moroni (2016) 
have solved the case for two-action, multiple-player, aggregative GSS with overlapping dominance regions.

We extend the global games method to multiple-player, multiple-action, monotone games, which include GSC, GSS, and arbitrary combinations of the two. In particular, our model encompasses the case in which all players exhibit strategic complements, and the more recent attempts to include strategic substitutes, and extends both to allow for any combination of the two. Moreover, in contrast to earlier work, we provide greater generality by requiring only that a player has strategic complements or substitutes in a given state. This allows for cases in which a player exhibits strategic complements in one state and strategic substitutes in another state.

Our approach is to directly extend the original $2 \times 2$ framework of CvD by drawing on common order properties present in both GSC and GSS, and by using the notion of $p$-dominance (a natural extension of risk dominance to multiple-player, multiple-action cases, following Morris, Rob, and Shin (1995) and Kajii and Morris (1997)), and by continuing to use dominance solvability as the solution concept. This helps to subsume earlier results as natural special cases.

By focusing on these core analytical components of the global games method, we are able to dispense with several additional assumptions introduced in work since CvD. In particular, we use only one dominance region (either a lower dominance region or an upper dominance region) as compared to two dominance regions (both lower and upper dominance region), common in work since CvD. Moreover, we do not require state monotonicity assumptions on preferences present in both FMP and Harrison and JaraMoroni (2016). Our results apply to non-aggregative games, an arbitrary finite number of players, and to cases with strategic heterogeneity (arbitrary combinations of strategic complements and strategic substitutes).

Our main result has the same flavor as CvD. For a profile of actions that is a Nash equilibrium in a complete information game that is embedded in a global game, if an 
open interval intersects the dominance region for this profile of actions and on that open interval, the profile satisfies a p-dominance condition, then on the whole interval, for sufficiently small noise, this is the unique profile that survives iterated elimination of strictly dominated strategies.

As earlier work has shown, when we move away from two players or from strategic complements, a common complication in applying the global games method is the information requirement inherent in belief formation and its impact on the contagion argument used in equilibrium selection. Indeed, we may view the additional assumptions (such as state monotonicity, or overlapping dominance regions, or aggregative structure) in work since $\mathrm{CvD}$ as devices to manage these complications in more general environments. We show that our $p$-dominance condition is an alternative sufficient condition, it is natural (in terms of the original motivation of risk dominance in $\mathrm{CvD}$ ) and it helps to generalize CvD with fewer additional assumptions.

As previous work has shown, the informational complexity of the global games method increases with the number of players and with strategic heterogeneity. In our case, this manifests by making the $p$-dominance condition more restrictive as the number of players increases. This is the direct result of the requirement that each player must keep track of the distribution of signals for all her opponents, and then the actions that opponents may choose to play for each signal they receive.

As extensions of our main result, we show that if the probabilistic burden involved in formulating conditional beliefs for every single opponent is relaxed, then the $p$-dominance condition is relaxed as well, and the applicability of our result increases correspondingly.

This is formalized by considering groups of opponents who receive the same signal. ${ }^{1}$ One motivation may be that some groups of players may naturally share the same signal. Another motivation for this approach is the idea of cognitive shortcuts from social

\footnotetext{
${ }^{1}$ Notably, we do not restrict payoffs for players in a group, allowing the same level of heterogeneity in player payoffs as earlier.
} 
psychology and experimental economics. In other words, keeping track of beliefs for every opponent is a cognitively daunting task when the number of opponents is large. In this case, decision makers may employ a cognitive shortcut by exhibiting "stereotyping behavior", which leads them to infer more correlation about opponents' types than would be present if they were to correctly form beliefs for each player separately (confer Macrae and Bodenhausen (2000) and Healy (2007)). We include results for both cases.

Formally, we postulate a partition of players into groups, and each player in the same group receives the same signal. As one case, we consider a partition of players into finitely many groups, consistent with a specified collection of players who share the same signal, and as another case, we consider partitions that may be different for different players, capturing the idea of hetergeneous stereotyping behavior or differing cognitive behavior. In both cases, the $p$-dominance condition for each player depends on number of groups in the relevant partition rather than the (larger) total number of players, thereby making it easier to hold. In the extreme case of discrete partitions, we are back to our main result for finitely many players. In the other extreme case of only two groups ("me" and "others") in a partition, we recover the full power of the two-player result, independent of the number of players. This is the weakest possible $p$-dominance requirement.

The paper is organized as follows. Section 2 formalizes a model for monotone global games and presents the main result. Section 3 gives some illustrative examples. Section 4 introduces group partitions which helps to relax the $p$-dominance condition and generalizes the main result. It also includes additional examples.

\section{Monotone Global Games}

The global games method is used to resolve the issue of multiple equilibria that arise in strategic situations by relaxing the assumption of common knowledge of an underlying parameter. Consider a complete information game with multiple equilibria at a certain 
parameter $x \in X_{0}$. We may view $x$ as the underlying payoff relevant state of the environment in which the game is played; for example, it may signify the strength of the economy in a game of speculative attack on a currency, or it may signify profitability of an investment opportunity in an investment game by two potential partners. Suppose there is some uncertainty about $x$. We add an information structure to formalize this uncertainty and study serially undominated strategies in the corresponding Bayesian game. We seek conditions under which for arbitrarily small amount of uncertainty, there is a unique Bayesian serially undominated strategy profile $s$, and then determine which action profile $s(x)$ is chosen at parameter $x \in X_{0}$. This provides a method to resolve the issue of multiple equilibria at $x$ in the complete information setting. A central feature in this approach is a contagion argument that strictly dominant actions in particular states can infect behavior in states further away in which actions are no longer strictly dominant.

Definition 1. A global game is a collection $\Gamma=\left\{\left(A_{i}, u_{i}\right)_{i=1}^{I}, X_{0},\left(\phi_{i}\right)_{i=1}^{I}, f, v\right\}$, in which

1. There are finitely many players $\mathcal{I}=\{1,2, \ldots, I\}$, and for each player $i \in \mathcal{I}$, there is a finite and linearly ordered set of actions $A_{i}$. The set of profiles of actions is denoted $A=\underset{i \in \mathcal{I}}{\times} A_{i}$ with common element $a \in A$.

2. There are payoff relevant states of the world $X_{0}=\left[\underline{x}_{0}, \bar{x}_{0}\right] \subset \mathbb{R}$. Each player's payoff is written $u_{i}: A \times X_{0} \rightarrow \mathbb{R}$, and is assumed to be continuous in $x$. When necessary, for $x \leq \underline{x}_{0}$, let $u_{i}(\cdot, x)=u_{i}\left(\cdot, \underline{x}_{0}\right)$ and for $x \geq \bar{x}_{0}$, let $u_{i}(\cdot, x)=u_{i}\left(\cdot, \bar{x}_{0}\right){ }^{2}$

3. For each $i \in \mathcal{I}$, player $i$ does not observe the true payoff state $x$, only a noisy signal

$$
x_{i}=x+v \epsilon_{i}
$$

where the $\epsilon_{i}$ are i.i.d and drawn from $[-1,1]$ according to an atomless, continuous, and symmetric density $\phi_{i}$, and $v>0$ is a scale factor. The state $x$ is realized with

\footnotetext{
${ }^{2}$ Extending payoffs beyond $\left[\underline{x}_{0}, \bar{x}_{0}\right]$ is done for notational convenience. This does not imply existence of dominance regions, which are defined below.
} 
density $f$, where $f$ is a continuous density with connected support, the interior of which contains $X_{0}$. Moreover, the $\left(\phi_{i}\right)_{i=1}^{I}$ and $f$ are assumed jointly independent.

In the analysis below, it will be useful to define the following. For player $i \in \mathcal{I}$, state $x \in \mathbb{R}$, and profile of opponents actions $a_{-i}$, the marginal benefit to player $i$ of playing action $\hat{a}_{i}$ over $\tilde{a}_{i}$ at $x$ is $\Delta u_{i}\left(\hat{a}_{i}, \tilde{a}_{i}, a_{-i}, x\right)=u_{i}\left(\hat{a}_{i}, a_{-i}, x\right)-u_{i}\left(\tilde{a}_{i}, a_{-i}, x\right)$.

A monotone global game is defined as follows. For player $i \in \mathcal{I}$ and state $x \in \mathbb{R}$, player $i$ has strategic complements at $x$, if $u_{i}$ has increasing differences in $\left(a_{i} ; a_{-i}\right)$ at $x$. That is, for every $\hat{a}_{i}>\tilde{a}_{i}, u_{i}\left(\hat{a}_{i}, \cdot, x\right)-u_{i}\left(\tilde{a}_{i}, \cdot, x\right)$ is (weakly) increasing in $a_{-i}{ }^{3}$ In other words, $\Delta u_{i}\left(\hat{a}_{i}, \tilde{a}_{i}, a_{-i}, x\right)$ is (weakly) increasing in $a_{-i}$. Similarly, player $i$ has strategic substitutes at $x$, if for every $\hat{a}_{i}>\tilde{a}_{i}, \Delta u_{i}\left(\hat{a}_{i}, \tilde{a}_{i}, a_{-i}, x\right)$ is (weakly) decreasing in $a_{-i}$. A monotone global game is a global game in which for every $i \in \mathcal{I}$ and every $x \in \mathbb{R}$, either player $i$ has strategic complements at $x$ or strategic substitutes at $x$.

Notice that this formulation is more flexible than previous works, for example, Frankel, Morris, and Pauzner (2003) and Harrison and Jara-Moroni (2016) in that our framework allows for each player to be either a complements or a substitutes player, and thus generalizes the extreme cases when all players are either one or the other. Moreover, we do not require, for example, that a player is a substitutes or complements player at all states $x$, only that they are one or the other at each $x$. That is, player $i$ may be a complements player at $x$ but a substitutes player at some other $x^{\prime}$.

We do require that a player $i$ is a complements or substitutes player with respect to all their opponents in the sense that the definition requires weakly increasing (or decreasing) differences in $a_{-i}$, the profile of actions of all opponents. In particular, at a given state $x$, we do not consider a player having increasing differences with respect to actions of some opponents and decreasing differences with respect to actions of other opponents.

Furthermore, notice that continuity of $u_{i}$ in $x$ implies that a player does not switch from having strategic substitutes to strategic complements discontinuously in the sense

\footnotetext{
${ }^{3}$ The partial order on finite-dimensional Euclidean space is the product partial order, as usual.
} 
that if $\left(x^{n}\right)$ is a sequence converging to $x$ and player $i$ has strategic substitutes along the sequence, then player $i$ has strategic substitutes at $x$, and similarly for strategic complements.

Dominance regions are defined as follows. Consider a profile of actions $a^{*}=\left(a_{1}^{*}, \ldots, a_{I}^{*}\right)$. For player $i$, the dominance region for $a_{i}^{*}$, denoted $D_{i}^{a_{i}^{*}}$, is the set of states where $a_{i}^{*}$ is strictly dominant for player $i$. That is,

$$
D_{i}^{a_{i}^{*}}=\left\{x \in \mathbb{R} \mid \forall a_{i} \neq a_{i}^{*}, \forall a_{-i}, \Delta u_{i}\left(a_{i}^{*}, a_{i}, a_{-i}, x\right)>0\right\} .
$$

A global game has a lower dominance region for $a^{*}$ at $\underline{x} \in \mathbb{R}$, if there is $i$ such that for every $x \leq \underline{x}, x \in \bigcap_{j \neq i} D_{j}^{a_{j}^{*}}$. In other words, a global game has a lower dominance region at $\underline{x}$ if there is $i$ such that $(-\infty, \underline{x}] \subset \bigcap_{j \neq i} D_{j}^{a_{j}^{*}}$. Notice that we require strict dominance for $I-1$ players only. Similarly, a global game has an upper dominance region for $a^{*}$ at $\bar{x} \in \mathbb{R}$, if there is $i$ such that for every $x \geq \underline{x}, x \in \bigcap_{j \neq i} D_{j}^{a_{j}^{*}}$. Finally, a global game has $\boldsymbol{a}$ dominance region for $a^{*}$, if the global game has either a lower dominance region for $a^{*}$ at $\underline{x}$, or an upper dominance region for $a^{*}$ at $\bar{x}$, for some $\underline{x}, \bar{x} \in \mathbb{R}$.

Belief formation is described as follows. Let $\tilde{\phi}_{i}^{v}$ be the density of signal errors $x_{i}-x$. Therefore, player $i$ 's beliefs over the signals $x_{-i}$ of opponents after observing $x_{i}$ are given by

$$
f_{i}\left(x_{-i} \mid x_{i}, v\right)=\frac{\int_{\mathbb{R}} f(x) \tilde{\phi}_{i}^{v}\left(x_{i}-x\right) \prod_{j \neq i} \tilde{\phi}_{j}^{v}\left(x_{j}-x\right) d m(x)}{\int_{\mathbb{R}} \int_{\mathbb{R}^{I-1}} f(x) \tilde{\phi}_{i}^{v}\left(x_{i}-x\right) \prod_{j \neq i} \tilde{\phi}_{j}^{v}\left(x_{j}-x\right) d m_{I-1}\left(x_{-i}\right) d m(x)},
$$

where $m$ is Lebesgue measure on $\mathbb{R}$ and $m_{I-1}$ is Lebesgue measure on $\mathbb{R}^{I-1}$. For notational convenience, beliefs are extended to $\mathbb{R}^{I-1}$, with the convention that if denominator is zero, set $f_{i}\left(x_{-i} \mid x_{i}, v\right)=0$. Let's denote the cdf by $F_{i}^{v}\left(x_{-i} ; x_{i}\right),{ }^{4}$ and the corresponding probability measure (for each Borel set $\left.\beta \subset \mathbb{R}^{I-1}\right)$ by $\mu_{i}\left(\beta \mid x_{i}, v\right)=\int_{\beta} f_{i}\left(x_{-i} \mid x_{i}, v\right) d m_{I-1}\left(x_{-i}\right)$.

Strategies are as follows. A strategy (or Bayesian strategy) for player $i$ is a Borel measurable function $s_{i}: \mathbb{R} \rightarrow A_{i}$, and the strategy space for player $i$ is the collection of

\footnotetext{
${ }^{4}$ Notice that support of this distribution is contained in $\left[x_{i}-2 v, x_{i}+2 v\right]^{I-1}$, because for $j \neq i$, $x_{j}=x+v \epsilon_{j}=x_{i}-v \epsilon_{i}+v \epsilon_{j}$, and the errors are distributed independently.
} 
all such strategies, denoted $\mathcal{S}_{i}$. Having observed signal $x_{i}$, the expected payoff to player $i$ from playing action $a_{i} \in A_{i}$ against a profile of opponents strategies $s_{-i} \in \times_{j \neq i} \mathcal{S}_{j}$ is then given by

$$
\pi_{i}^{v}\left(a_{i}, s_{-i}, x_{i}\right)=\int_{\mathbb{R}^{I-1}} u_{i}\left(a_{i}, s_{-i}\left(x_{-i}\right), x_{i}\right) d \mu_{i}\left(x_{-i} \mid x_{i}, v\right) .
$$

As $u_{i}$ is continuous in $x$, and each player's action set is finite, it follows that each profile $s_{-i}$ consists of uniformly bounded and measurable functions, and hence, $\pi_{i}^{v}\left(a_{i}, s_{-i}, x_{i}\right)$ is well-defined and jointly continuous in $\left(s_{-i}, x_{i}\right)$. The (expected) marginal benefit to player $i$ of playing action $\hat{a}_{i}$ over $\tilde{a}_{i}$ at $x_{i}$ when opponents are playing $s_{-i}$ is given by $\Delta \pi_{i}^{v}\left(\hat{a}_{i}, \tilde{a}_{i}, s_{-i}, x_{i}\right)=\pi_{i}^{v}\left(\hat{a}_{i}, s_{-i}, x_{i}\right)-\pi_{i}^{v}\left(\tilde{a}_{i}, s_{-i}, x_{i}\right)$, and this is jointly continuous in $\left(s_{-i}, x_{i}\right)$ as well. A profile of strategies is denoted $s$, where $s=\left(s_{1}, \ldots, s_{I}\right)$.

With these definitions, here's a glimpse of a main result (theorem 1 below) in the paper. Let $\Gamma$ be a monotone global game with a dominance region for a profile of actions $a^{*}$, and let $\hat{X} \subset X_{0}$ be a set on which a $p$-dominance condition (details are given below) holds. For every open interval $\mathcal{O} \subset \hat{X}$ such that either $\mathcal{O} \cap(-\infty, \underline{x}] \neq \emptyset$, or $\mathcal{O} \cap[\bar{x}, \infty) \neq \emptyset$ (depending on which dominance region exists), $\forall x \in \mathcal{O}, \exists \hat{v}>0, \forall v \in(0, \hat{v}]$, for every serially undominated strategy profile $s, s(x)=a^{*}$. We prove this result using several steps along the way, as formalized next.

\subsection{Simple Global Game}

In order to establish a global games prediction in a general global game $\Gamma$, our approach is to first establish a prediction in the corresponding simple global game and then extend the result to the general global game. The simple global game associated with global game $\Gamma$, denoted $\Gamma^{*}$, is the same as $\Gamma$ but with the following two assumptions about distributions. First, the prior over states is uniform, denoted $f^{*}$, and contains $\left[\underline{x}_{0}-4 v, \bar{x}_{0}+4 v\right]$ in its

support. Second, errors are distributed according to a uniform distribution, denoted $\phi_{i}^{*}$. Notice that $f^{*}$ is constant and positive on its entire support, and therefore, player $i$ 's 
conditional beliefs about $x_{-i}$ after receiving signal $x_{i}$ are given by ${ }^{5}$

$$
f_{i}^{*}\left(x_{-i} \mid x_{i}, v\right)=\int_{\mathbb{R}} \tilde{\phi}_{i}^{v, *}\left(x_{i}-x\right) \prod_{j \neq i} \tilde{\phi}_{j}^{v, *}\left(x_{j}-x\right) d m(x) .
$$

Let's denote the cdf by $F_{i}^{*}\left(x_{-i} ; x_{i}, v\right)$, and let $\mu_{i}^{*}\left(\cdot \mid x_{i}, v\right)$ denote the corresponding probability measure.

Proposition 1 below gives two results which are useful to extend results from a simple global game to the general global game. The first, due to Carlsson and van Damme (1993), shows that in a 2-player simple global game, player $i$ 's belief about the signal $x_{j}$ received by her opponent is determined exactly by player $j$ 's belief about the signal $x_{i}$ received by player $i$. The second is similar in nature, and shows that in the multiple player case, there is a lower bound on player $i$ 's belief that opponents have received a signal lower than hers. To establish notation, if player $i$ receives signal $x_{i}$, let $x_{i} \mathbf{1}_{I-1}$ be the $(I-1)$-dimensional vector with the constant number $x_{i}$ in each component.

Proposition 1. Suppose $\Gamma^{*}$ is a simple global game.

1. (CvD, 1993) Suppose $I=2$. If for every player $i, B\left(x_{i}, 2 v\right) \subset X_{0}$, then

$$
F_{1}^{*}\left(x_{2} \mid x_{1}, v\right)+F_{2}^{*}\left(x_{1} \mid x_{2}, v\right)=1 .
$$

2. Suppose $I \geq 2$. If for every player $i, B\left(x_{i}, 2 v\right) \subset X_{0}$, then

$$
F_{i}^{*}\left(x_{i} \mathbf{1}_{I-1} \mid x_{i}, v\right) \geq \frac{1}{I}
$$

Proof. See appendix.

The next result, due to Frankel, Morris, and Pauzner (2003), establishes a key relationship between a global game $\Gamma$ and its simple counterpart $\Gamma^{*}$. That is, as noise $v>0$ becomes small, beliefs in $\Gamma$ approach those in $\Gamma^{*}$. We will measure the distance between two probability measures over the signals received by opponents by the sup

\footnotetext{
${ }^{5}$ We define $\tilde{\phi}_{i}^{v, *}=\frac{1}{v} \phi_{i}^{*}$ to give the corresponding pdf for $x_{i}-x$.
} 
norm. Let $\triangle\left(\mathbb{R}^{I-1}\right)$ be the set of all probability measures on Borel sets of $\mathbb{R}^{I-1}$, and for $\mu, \nu \in \triangle\left(\mathbb{R}^{I-1}\right), \mu$ is $\varepsilon$-close to $\nu$, if it is in the $\varepsilon$-neighborhood of $\nu$, defined as

$$
B(\nu, \varepsilon)=\left\{\mu \in \triangle\left(\mathbb{R}^{I-1}\right) \mid \sup _{\beta}(|\mu(\beta)-\nu(\beta)|) \leq \varepsilon\right\},
$$

where the supremum is over Borel sets $\beta \subset \mathbb{R}^{I-1}$. We then have the following:

Proposition 2. (FMP, Lemma A2) Suppose that $\left[\underline{x}_{0}-2 v, \bar{x}_{0}+2 v\right]$ is contained within the interior of the support of $f$ for some $v>0$. Then, for every $\varepsilon>0$, there exists $\bar{v}>0$ such that for each $x \in X_{0}$, and each $v \leq \bar{v}, \mu_{i}(\cdot \mid x, v) \in B\left(\mu_{i}^{*}(\cdot \mid x, v), \varepsilon\right)$.

Proof. See lemma A2 in Frankel, Morris, and Pauzner (2003).

In particular, proposition 2 implies that in the case of $I=2$, for all $\epsilon>0$, there exists $\bar{v}>0$ such that for all $v<\bar{v}$, and all $x_{1}, x_{2}$ such that $B\left(x_{i}, 2 v\right) \subset X_{0}$ for $i=1,2$,

$$
F_{1}\left(x_{2} \mid x_{1}, v\right)+F_{2}\left(x_{1} \mid x_{2}, v\right) \geq 1-\epsilon
$$

Likewise, for $I \geq 2$, an analogous argument yields for all $\epsilon>0$, there exists $\bar{v}>0$ such that for all $v<\bar{v}$, and all $x_{i}$ such that $B\left(x_{i}, 2 v\right) \subset X_{0}$,

$$
F_{i}\left(x_{i} \mathbf{1}_{I-1} \mid x_{i}, v\right) \geq \frac{1}{I}-\epsilon
$$

These will be useful to extend the results of proposition 1 to the general global game $\Gamma$, where the assumptions of a uniform prior and uniform errors are dropped.

\section{$2.2 \quad$ Iterative Procedure}

We now proceed to describe the iterative procedure which will lead to a serially undominated Bayesian strategy. Consider a global game $\Gamma$ and a profile of actions $a=$ $\left(a_{1}, \ldots, a_{I}\right) \in A$. 
For each player $i$, and for each $a_{i} \in A_{i}$, let $\mathcal{X}_{i, a_{i}}^{v, 0}=\emptyset$, and let $\mathcal{S}_{i}^{v, 0}=\mathcal{S}_{i}$, and define inductively, for $n \geq 1$,

$$
\begin{aligned}
\mathcal{X}_{i, a_{i}}^{v, n} & =\left\{x_{i} \in \mathbb{R} \mid \forall a_{i}^{\prime} \neq a_{i}, \forall s_{-i} \in \mathcal{S}_{-i}^{v, n-1}, \Delta \pi_{i}^{v}\left(a_{i}, a_{i}^{\prime}, s_{-i}, x_{i}\right)>0\right\} \text { and } \\
\mathcal{S}_{i}^{v, n} & =\left\{s_{i} \in \mathcal{S}_{i}^{v, n-1}\left|\forall a_{i} \in A_{i}, s_{i}\right|_{\mathcal{X}_{i, a_{i}}^{v, n}}=a_{i}\right\} .
\end{aligned}
$$

The set $\mathcal{X}_{i, a_{i}}^{v, n}$ is the set of all $x_{i}$ where $a_{i}$ is (strictly) dominant for player $i$ when opponent strategies are in $\mathcal{S}_{i}^{v, n-1}$. The set $\mathcal{S}_{i}^{v, n}$ are all strategies that play $a_{i}$ on $\mathcal{X}_{i, a_{i}}^{v, n}$. Using induction, it is easy to check that for every $a_{i} \in A_{i}$ and $n \geq 0, \mathcal{X}_{i, a_{i}}^{v, n} \subset \mathcal{X}_{i, a_{i}}^{v, n+1}$, and for every $n \geq 0, \mathcal{S}_{i}^{v, n+1} \subset \mathcal{S}_{i}^{v, n}$. Let $\mathcal{X}_{i, a_{i}}^{v}=\bigcup_{n=0}^{\infty} \mathcal{X}_{i, a_{i}}^{v, n}$ and $\mathcal{S}_{i}^{v}=\bigcap_{n=0}^{\infty} \mathcal{S}_{i}^{v, n}$.

As $A_{i}$ is a finite linearly ordered set, let $\underline{a}_{i}$ denote the smallest element of $A_{i}$ and $\bar{a}_{i}$ the largest. For $n \geq 0$, consider the strategies $\underline{s}_{i}^{v, n}$ and $\bar{s}_{i}^{v, n}$ given by

$$
\underline{s}_{i}^{v, n}\left(x_{i}\right)=\left\{\begin{array}{ll}
a_{i} & \text { if } x_{i} \in \mathcal{X}_{i, a_{i}}^{v, n} \\
\underline{a}_{i} & \text { if } x_{i} \notin \bigcup_{a_{i} \in A_{i}} \mathcal{X}_{i, a_{i}}^{v, n}
\end{array} \quad \text { and } \quad \bar{s}_{i}^{v, n}\left(x_{i}\right)= \begin{cases}a_{i} & \text { if } x_{i} \in \mathcal{X}_{i, a_{i}}^{v, n} \\
\bar{a}_{i} & \text { if } x_{i} \notin \bigcup_{a_{i} \in A_{i}} \mathcal{X}_{i, a_{i}}^{v, n} .\end{cases}\right.
$$

Notice that for every $n \geq 0, \underline{s}_{i}^{v, n} \leq \bar{s}_{i}^{v, n}$. Combined with the fact that $\mathcal{X}_{i, a_{i}}^{v, n} \subset \mathcal{X}_{i, a_{i}}^{v, n+1}$, it follows that for every $n \geq 0, \underline{s}_{i}^{v, n} \leq \underline{s}_{i}^{v, n+1} \leq \bar{s}_{i}^{v, n+1} \leq \bar{s}_{i}^{v, n}$. Let $\underline{s}_{i}^{v}$ be defined by $\underline{s}_{i}^{v}\left(x_{i}\right)=\lim _{n \rightarrow \infty} \underline{s}_{i}^{v, n}\left(x_{i}\right)$ and $\bar{s}_{i}^{v}$ be defined by $\bar{s}_{i}^{v}\left(x_{i}\right)=\lim _{n \rightarrow \infty} \bar{s}_{i}^{v, n}\left(x_{i}\right)$. Moreover, for each $n$, let $\left[\underline{s}_{i}^{v, n}, \bar{s}_{i}^{v, n}\right]$ be the order interval of functions given by

$$
\left[\underline{s}_{i}^{v, n}, \bar{s}_{i}^{v, n}\right]=\left\{s_{i} \in \mathcal{S}_{i} \mid \forall x_{i} \in \mathbb{R}, \underline{s}_{i}^{v, n}\left(x_{i}\right) \leq s_{i}\left(x_{i}\right) \leq \bar{s}_{i}^{v, n}\left(x_{i}\right)\right\}
$$

and define $\left[\underline{s}_{i}^{v}, \bar{s}_{i}^{v}\right]$ similarly.

Proposition 3. Let $\Gamma$ be a global game.

1. For every $n \geq 0, \mathcal{S}_{i}^{v, n}=\left[\underline{s}_{i}^{v, n}, \bar{s}_{i}^{v, n}\right]$

2. $\mathcal{S}_{i}^{v}=\left[\underline{s}_{i}^{v}, \bar{s}_{i}^{v}\right]=\left\{s_{i} \in \mathcal{S}_{i}\left|\forall a_{i} \in A_{i}, s_{i}\right|_{\mathcal{X}_{i, a_{i}}^{v}}=a_{i}\right\}$

Proof. To prove statement (1), notice that for $n=0$, the statement is trivially true. Moreover, for $n \geq 1, \mathcal{S}_{i}^{v, n} \subset\left[\underline{s}_{i}^{v, n}, \bar{s}_{i}^{v, n}\right]$ is trivially true. In the other direction, consider 
$s_{i} \in\left[\underline{s}_{i}^{v, n}, \bar{s}_{i}^{v, n}\right], a_{i} \in A_{i}$, and $x_{i} \in \mathcal{X}_{i, a_{i}}^{v, n}$. Then $a_{i}=\underline{s}_{i}^{v, n}\left(x_{i}\right) \leq s_{i}\left(x_{i}\right) \leq \bar{s}_{i}^{v, n}\left(x_{i}\right)=a_{i}$, whence $s_{i}\left(x_{i}\right)=a_{i}$. Therefore, $s_{i} \in \mathcal{S}_{i}^{v, n}$.

To prove the first equality in statement (2), notice that $\bigcap_{n=0}^{\infty}\left[\underline{s}_{i}^{v, n}, \bar{s}_{i}^{v, n}\right] \supset\left[\underline{s}_{i}^{v}, \bar{s}_{i}^{v}\right]$, because for every $n \geq 0,\left[\underline{s}_{i}^{v, n}, \bar{s}_{i}^{v, n}\right] \supset\left[\underline{s}_{i}^{v}, \bar{s}_{i}^{v}\right]$. In the other direction, suppose $s_{i} \in \bigcap_{n=0}^{\infty}\left[\underline{s}_{i}^{v, n}, \bar{s}_{i}^{v, n}\right]$. Then for all $n \geq 0, \underline{s}_{i}^{v, n} \leq s_{i} \leq \bar{s}_{i}^{v, n}$, whence for every $x_{i} \in \mathbb{R}, \underline{s}_{i}^{v}\left(x_{i}\right)=\lim _{n \rightarrow \infty} \underline{s}_{i}^{v, n}\left(x_{i}\right) \leq$ $s_{i}\left(x_{i}\right) \leq \lim _{n} \bar{s}_{i}^{v, n}\left(x_{i}\right)=\bar{s}_{i}^{v}\left(x_{i}\right)$. Consequently, $\mathcal{S}_{i}^{v}=\bigcap_{n=0}^{\infty} \mathcal{S}_{i}^{v, n}=\left[\underline{s}_{i}^{v}, \bar{s}_{i}^{v}\right]$.

To prove the second equality in statement (2), suppose first that $s_{i} \in \mathcal{S}_{i}^{v}=\bigcap_{n=0}^{\infty} \mathcal{S}_{i}^{v, n}$. Fix arbitrarily $a_{i} \in A_{i}$ and $x \in \mathcal{X}_{i, a_{i}}^{v}=\bigcup_{n=0}^{\infty} \mathcal{X}_{i, a_{i}}^{v, n}$. Then $\exists N \geq 1$ such that for $n \geq N$, $x \in \mathcal{X}_{i, a_{i}}^{v, n}$. Moreover, $s_{i} \in \mathcal{S}_{i}^{v, N-1}$. Therefore, $s_{i}(x)=a_{i}$, as desired. In the other direction, suppose $s_{i} \in \mathcal{S}_{i}$ is such that for all $a_{i} \in A_{i},\left.s_{i}\right|_{\mathcal{X}_{i, a_{i}}^{v}}=a_{i}$. Fix arbitrarily $n \geq 0, a_{i} \in A_{i}$, and $x_{i} \in \mathcal{X}_{i, a_{i}}^{v, n}$. As $\mathcal{X}_{i, a_{i}}^{v, n} \subset \mathcal{X}_{i, a_{i}}^{v}, s_{i}\left(x_{i}\right)=a_{i}$. It follows that for every $n \geq 0, s_{i} \in \mathcal{S}_{i}^{v, n}$.

Notice that the set of strategies $\mathcal{S}^{v}$ resulting from the iterative procedure above is more inclusive than the set of serially undominated strategies. For a player $i$, signal $x_{i} \in \mathbb{R}$, and set of opponents strategies $S_{-i}^{\prime} \subseteq \mathcal{S}_{-i}$, let

$$
\mathcal{U R}_{i}^{v}\left(S_{-i}^{\prime}, x_{i}\right)=\left\{a_{i} \in A_{i} \mid \forall a_{i}^{\prime} \neq a_{i}, \exists s_{-i} \in S_{-i}^{\prime}, \triangle \pi_{i}^{v}\left(a_{i}, a_{i}^{\prime}, s_{-i}, x_{i}\right) \geq 0\right\}
$$

be the set of player $i$ 's undominated actions at $x_{i}$ to opponents strategies in $S_{-i}^{\prime}$, and let $\mathcal{U R}_{i}^{v}\left(S_{-i}^{\prime}\right)=\left\{s_{i} \in \mathcal{S}_{i} \mid \forall x_{i} \in X_{i}, s_{i}(x) \in \mathcal{U R}_{i}^{v}\left(S_{-i}^{\prime}, x_{i}\right)\right\}$ be the set of player $i$ 's undominated responses to opponent strategies in $S_{-i}^{\prime}$, and let $\mathcal{U} \mathcal{R}^{v}\left(S^{\prime}\right)=\left(\mathcal{U} \mathcal{R}_{i}^{v}\left(S_{-i}^{\prime}\right)\right)_{i \in \mathcal{I}}$ be the collection of such sets for each player $i$. Serially undominated strategies are then defined through the usual iterative procedure: For $n=0$, let $\mathcal{U} \mathcal{R}^{v, 0}=\mathcal{S}$, and for each $n \geq 1$, let $\mathcal{U} \mathcal{R}^{v, n}=\mathcal{U} \mathcal{R}^{v}\left(\mathcal{U} \mathcal{R}^{v, n-1}\right)$. The set of serially undominated strategies is given by $\mathcal{S U}^{v}=\bigcap_{n=0}^{\infty} \mathcal{U} \mathcal{R}^{v, n}$

It is easy to see that for each $n \geq 0, \mathcal{U} \mathcal{R}^{v, n} \subset \mathcal{S}^{v, n}$, and hence, $\mathcal{S U}^{v} \subset \mathcal{S}^{v}$. Therefore, if we prove that every $s \in \mathcal{S}^{v}$ selects a profile of actions $a \in A$ at some $x$, then we can conclude that every serially undominated strategy does so as well, and hence $a$ is the global games prediction at $x$. 


\subsection{Characterizing $\mathcal{X}_{i, a_{i}}^{v}$}

We now show through propositions 4 and 5 how strategic substitutes and complements allow us to characterize the sets $\mathcal{X}_{i, a_{i}}^{v}$.

Proposition 4. Let $\Gamma$ be a monotone global game and $a^{*}$ a profile of actions.

1. Suppose player $i$ has strategic substitutes at $x_{i}$. Then the following are equivalent.

(a) $x_{i} \in \mathcal{X}_{i, a_{i}^{*}}^{v}$

(b) $\forall a_{i}^{\prime}<a_{i}^{*}, \Delta \pi_{i}^{v}\left(a_{i}^{*}, a_{i}^{\prime}, \bar{s}_{-i}^{v}, x_{i}\right)>0$, and $\forall a_{i}^{\prime}>a_{i}^{*}, \Delta \pi_{i}^{v}\left(a_{i}^{*}, a_{i}^{\prime}, \underline{s}_{-i}^{v}, x_{i}\right)>0$.

2. Suppose player $i$ has strategic complements at $x_{i}$. Then the following are equivalent.

(a) $x_{i} \in \mathcal{X}_{i, a_{i}^{*}}^{v}$

(b) $\forall a_{i}^{\prime}>a_{i}^{*}, \Delta \pi_{i}^{v}\left(a_{i}^{*}, a_{i}^{\prime}, \bar{s}_{-i}^{v}, x_{i}\right)>0$, and $\forall a_{i}^{\prime}<a_{i}^{*}, \Delta \pi_{i}^{v}\left(a_{i}^{*}, a_{i}^{\prime}, \underline{s}_{-i}^{v}, x_{i}\right)>0$.

3. $\mathcal{X}_{i, a_{i}^{*}}^{v}=\left\{x_{i} \in \mathbb{R} \mid \forall s_{-i} \in\left[\underline{s}_{-i}^{v}, \bar{s}_{-i}^{v}\right], \forall a_{i}^{\prime} \neq a_{i}^{*}, \Delta \pi_{i}^{v}\left(a_{i}^{*}, a_{i}^{\prime}, s_{-i}, x_{i}\right)>0\right\}$.

Proof. To prove statement (1), suppose that $x_{i} \in \mathcal{X}_{i, a_{i}^{*}}^{v}$ Fix $a_{i}^{\prime}>a_{i}^{*}$. As $\mathcal{X}_{i, a_{i}^{*}}^{v}=$ $\bigcup_{n=0}^{\infty} \mathcal{X}_{i, a_{i}^{*}}^{v, n}$, there is $N$ such that for all $n \geq N, x_{i} \in \mathcal{X}_{i, a_{i}^{*}}^{v, n}$ Moreover, $\mathcal{S}_{i}^{v}=\bigcap_{n=0}^{\infty} \mathcal{S}_{i}^{v, n}$, and therefore, $\underline{s}_{-i}^{v}, \bar{s}_{-i}^{v} \in \mathcal{S}_{i}^{v, N-1}$, and using definition of $\mathcal{X}_{i, a_{i}^{*}}^{v, N}$, it follows that $\forall a_{i}^{\prime} \neq$ $a_{i}^{*}, \Delta \pi_{i}^{v}\left(a_{i}^{*}, a_{i}^{\prime}, \bar{s}_{-i}^{v}, x_{i}\right)>0$ and $\Delta \pi_{i}^{v}\left(a_{i}^{*}, a_{i}^{\prime}, \underline{s}_{-i}^{v}, x_{i}\right)>0$. The case of $a_{i}^{\prime}<a_{i}^{*}$ is similar.

Conversely, $\operatorname{suppose}(1)(\mathrm{b})$ is true and suppose that $x_{i} \notin \mathcal{X}_{i, a_{i}^{*}}^{v}$. Then for all $n \geq 1$, $x_{i} \notin \mathcal{X}_{i, a_{i}^{*}}^{v, n}$. That is, for all $n \geq 1$, there is $a_{i}^{n} \neq a_{i}^{*}$ and there is $s_{-i}^{n} \in \mathcal{S}_{-i}^{n-1}$ such that $\Delta \pi_{i}^{v}\left(a_{i}^{*}, a_{i}^{n}, s_{-i}^{n}, x_{i}\right) \leq 0$. As $A_{i}$ is finite, going to a subsequence and relabeling if necessary, let $a_{i}^{n}$ be constant at $\tilde{a}_{i} \neq a_{i}^{*}$. As case 1 , suppose $\tilde{a}_{i}<a_{i}^{*}$. Then for all $n \geq 1, \Delta \pi_{i}^{v}\left(a_{i}^{*}, \tilde{a}_{i}, \bar{s}_{-i}^{n}, x_{i}\right) \leq \Delta \pi_{i}^{v}\left(a_{i}^{*}, \tilde{a}_{i}, s_{-i}^{n}, x_{i}\right) \leq 0$, where the first weak inequality is a consequence of strategic substitutes. Moreover, by assumption, $\Delta \pi_{i}^{v}\left(a_{i}^{*}, \tilde{a}_{i}, \bar{s}_{-i}, x_{i}\right)>$ 0 , and thus by continuity in $s_{-i}$ we have that there is $N$ such that for all $n \geq N$, $\Delta \pi_{i}^{v}\left(a_{i}^{*}, \tilde{a}_{i}, \bar{s}_{-i}^{n}, x_{i}\right)>0$, a contradiction. The case when $\tilde{a}_{i}>a_{i}^{*}$, as well as the cases in statement (2) for strategic complements, are proven similarly. 
To prove statement (3), suppose player $i$ has strategic substitutes at $x_{i} \in \mathcal{X}_{i, a_{i}^{*}}^{v}$, and suppose $s_{-i} \in\left[\underline{s}_{-i}, \bar{s}_{-i}\right]$, and $a_{i}^{\prime}<a_{i}^{*}$. Then $0<\Delta \pi_{i}^{v}\left(a_{i}^{*}, a_{i}^{\prime}, \bar{s}_{-i}^{v}, x_{i}\right) \leq \Delta \pi_{i}^{v}\left(a_{i}^{*}, a_{i}^{\prime}, s_{-i}, x_{i}\right)$, where the strict inequality follows from statement (1) and the weak inequality is a consequence of strategic substitutes at $x_{i}$. The case where $a_{i}^{\prime}>a_{i}^{*}$ follows similarly. The reverse inclusion follows from statement (1). The case when player $i$ has strategic complements at $x_{i}$ follows similarly.

Proposition 5. Let $\Gamma$ be a monotone global game and $a^{*}$ a profile of actions.

1. For every player $i, \mathcal{X}_{i, a_{i}^{*}}^{v}$ is an open set.

2. For every player $i$ and for every $x \in \bigcap_{j \neq i} D_{j}^{a_{j}^{*}}$, if $a_{i}^{*}$ is player $i$ 's unique best response to $a_{-i}^{*}$ at $x$, then $\exists \bar{v}>0, \forall v \in[0, \bar{v}), B(x, 2 v) \subset \bigcap_{i \in \mathcal{I}} \mathcal{X}_{i, a_{i}^{*}}^{v}$.

Proof. Notice first that finiteness of every $A_{i}$ and continuity of $u_{i}$ in $x$ implies that $D_{i}^{a_{i}^{*}}$ is an open set. Moreover, for every $v>0, D_{i}^{a_{i}^{*}} \subset \mathcal{X}_{i, a_{i}^{*}}^{v}$.

For statement 1 , let $i \in \mathcal{I}, a_{i}^{*} \in A_{i}$, and $x_{i} \in \mathcal{X}_{i, a_{i}^{*}}^{v}$. Proposition 4 , statement 3 implies that $\forall a_{i}^{\prime} \neq a_{i}^{*}, \Delta \pi_{i}^{v}\left(a_{i}^{*}, a_{i}^{\prime}, \bar{s}_{-i}^{v}, x_{i}\right)>0$, and $\forall a_{i}^{\prime} \neq a_{i}^{*}, \Delta \pi_{i}^{v}\left(a_{i}^{*}, a_{i}^{\prime}, \underline{s}_{-i}^{v}, x_{i}\right)>0$. As $\Delta \pi_{i}^{v}$ is continuous in $x$, there is $\epsilon>0$ such that for each $x^{\prime} \in B\left(x_{i}, \epsilon\right), \forall a_{i}^{\prime} \neq a_{i}^{*}$, $\Delta \pi_{i}^{v}\left(a_{i}^{*}, a_{i}^{\prime}, \bar{s}_{-i}^{v}, x^{\prime}\right)>0$, and $\forall a_{i}^{\prime} \neq a_{i}^{*}, \Delta \pi_{i}^{v}\left(a_{i}^{*}, a_{i}^{\prime}, \underline{s}_{-i}^{v}, x^{\prime}\right)>0$. Applying statement 1 or 2 of proposition 4 , as the case may be at $x^{\prime}$, we conclude that $x^{\prime} \in \mathcal{X}_{i, a_{i}^{*}}^{v}$, as desired.

For statement 2, first notice that $x \in \bigcap_{j \neq i} D_{j}^{a_{j}^{*}} \subset \bigcap_{j \neq i} \mathcal{X}_{j, a_{j}^{*}}^{v}$ for all $v>0$, and also $\bigcap_{j \neq i} D_{j}^{a_{j}^{*}}$ is open. Therefore, there exists $\epsilon>0$ such that $B(x, 2 \epsilon) \subset \bigcap_{j \neq i} \mathcal{X}_{j, a_{j}^{*}}^{v}$ for all $v>0$. As $\Delta u_{i}$ is continuous in $x$ and $a_{i}^{*}$ is unique best response to $a_{-i}^{*}$ at $x$, we may further assume that for every $x^{\prime} \in B(x, 2 \epsilon)$, and for every $a_{i} \neq a_{i}^{*}, \Delta u_{i}\left(a_{i}^{*}, a_{i}, a_{-i}^{*}, x^{\prime}\right)>0$. Choose $\bar{v}>0$ so that $4 \bar{v}<\epsilon$ and let $v<\bar{v}$. Then, for each $x^{\prime} \in B(x, 2 v)$ and each $x^{\prime \prime} \in B\left(x^{\prime}, 2 v\right)$, we have that $d\left(x^{\prime \prime}, x\right) \leq d\left(x^{\prime \prime}, x^{\prime}\right)+d\left(x^{\prime}, x\right)<4 v<\epsilon$, so that for each $x^{\prime} \in B(x, 2 v)$, $B\left(x^{\prime}, 2 v\right) \subset B(x, \epsilon) \subset \bigcap_{j \neq i} \mathcal{X}_{j, a_{j}^{*}}^{v}$. Therefore, if player $i$ receives any signal $x^{\prime} \in B(x, 2 v)$, the support of $\mu_{i}\left(\cdot \mid x^{\prime}, v\right)$ lies entirely within $\bigcap_{j \neq i} \mathcal{X}_{j, a_{j}^{*}}^{v}$, so that for all $s_{-i} \in\left[\underline{s}_{-i}^{v}, \bar{s}_{-i}^{v}\right]$, and all 
$a_{i} \neq a_{i}^{*}$, we have

$$
\Delta \pi_{i}^{v}\left(a_{i}^{*}, a_{i}, s_{-i}, x^{\prime}\right)=\Delta u_{i}\left(a_{i}^{*}, a_{i}, a_{-i}^{*}, x^{\prime}\right)>0 .
$$

By proposition 4 , it follows that $x^{\prime} \in \mathcal{X}_{i, a_{i}^{*}}^{v}$, and therefore, $B(x, 2 v) \subset \mathcal{X}_{i, a_{i}^{*}}^{v} \cap \bigcap_{j \neq i} \mathcal{X}_{j, a_{j}^{*}}^{v}$

This proposition is useful to show when an action is consistent with a global games selection. In particular, $x \in \bigcap_{i \in \mathcal{I}} \mathcal{X}_{i, a_{i}^{*}}^{v} \Rightarrow \forall i, \forall s_{i} \in \mathcal{S}_{i}^{v}, s_{i}(x)=a_{i}^{*}$.

\subsection{Main Results}

In order to determine which equilibrium is chosen in the presence of uncertainty over payoff states, we will first need a way to quantify how dominant is an equilibrium profile in the presence of strategic uncertainty at a given state. In their $2 \times 2$ formulation, CvD employ the notion of risk dominance, and show that the risk dominant equilibrium will be the one that is selected in the global games procedure. In multiple-player, multiple-action games the natural notion is $p$-dominance.

In what follows, it will be helpful to relate player $i$ 's best response against $s_{-i}$ to her best response to the corresponding distribution of actions generated by $s_{-i}$. In order to formalize this connection, let's first define payoffs to a player from an action when others are playing a distribution over actions. Let $\mathcal{M}_{-i}$ denote the set of probability distributions over the actions of opponents of $i\left(\times_{j \neq i} A_{j}\right)$. The expected payoff to player $i$ from playing $a_{i}$ at $x_{i}$ against the distribution $\lambda_{-i} \in \mathcal{M}_{-i}$ is $\mathcal{U}_{i}\left(a_{i}, \lambda_{-i}, x_{i}\right)=\Sigma_{a_{-i}} \lambda_{-i}\left(a_{-i}\right) u_{i}\left(a_{i}, a_{-i}, x_{i}\right)$, and the expected marginal benefit of $\hat{a}_{i}$ over $\tilde{a}_{i}$ is $\Delta \mathcal{U}_{i}\left(\hat{a}_{i}, \tilde{a}_{i}, \lambda_{-i}, x_{i}\right)=\mathcal{U}_{i}\left(\hat{a}_{i}, \lambda_{-i}, x_{i}\right)-$ $\mathcal{U}_{i}\left(\tilde{a}_{i}, \lambda_{-i}, x_{i}\right)$. As $u_{i}$ is continuous in $x$ and each player's action set is finite, it follows that $\Delta \mathcal{U}_{i}$ is jointly continuous in $\left(\lambda_{-i}, x_{i}\right)$.

Consider the following connection. Given a profile of strategies for opponents of player $i, s_{-i}^{\prime} \in \mathcal{S}_{-i}$, define $\lambda_{-i}^{\prime} \in \mathcal{M}_{-i}$ as $\lambda_{-i}^{\prime}\left(a_{-i}\right)=\mu_{i}\left(\left\{s_{-i}^{\prime}=a_{-i}\right\} \mid x_{i}, v\right)$. In other words, $\lambda_{-i}^{\prime}\left(a_{-i}\right)$ is the probability that opponents of $i$ play $a_{-i}$ under $s_{-i}^{\prime}$, given player $i$ 's beliefs 
are $\mu_{i}\left(\cdot \mid x_{i}, v\right)$. In this case, for actions $a_{i}, a_{i}^{\prime}$ for player $i$,

$$
\begin{aligned}
\Delta \pi_{i}^{v}\left(a_{i}, a_{i}^{\prime}, s_{-i}^{\prime}, x_{i}\right) & =\int \Delta u_{i}\left(a_{i}, a_{i}^{\prime}, s_{-i}^{\prime}\left(x_{-i}\right), x_{i}\right) d \mu_{i}\left(x_{-i} \mid x_{i}, v\right) \\
& =\sum_{a_{-i}} \int \Delta u_{i}\left(a_{i}, a_{i}^{\prime}, a_{-i}, x_{i}\right) 1_{\left\{s_{-i}^{\prime}=a_{-i}\right\}} d \mu_{i}\left(x_{-i} \mid x_{i}, v\right) \\
& =\sum_{a_{-i}} \Delta u_{i}\left(a_{i}, a_{i}^{\prime}, a_{-i}, x_{i}\right) \lambda_{-i}^{\prime}\left(a_{-i}\right) \\
& =\Delta \mathcal{U}_{i}\left(a_{i}, a_{i}^{\prime}, \lambda_{-i}^{\prime}, x_{i}\right) .
\end{aligned}
$$

To generalize the notion of risk dominance, we use the notion of $p$-dominance, following Morris, Rob, and Shin (1995), and Kajii and Morris (1997). Let $a^{*} \in A$ be a profile of actions. For each player $i$ and for each $x \in \mathbb{R}$, let

$$
p_{i}^{a_{i}^{*}}(x)=\inf \left\{\xi \in[0,1] \mid \forall \lambda_{-i} \in \mathcal{M}_{-i}, \lambda_{-i}\left(a_{-i}^{*}\right) \geq \xi \Rightarrow \forall a_{i} \neq a_{i}^{*}, \Delta \mathcal{U}_{i}\left(a_{i}^{*}, a_{i}, \lambda_{-i}, x\right) \geq 0\right\} .
$$

Let's say that action $a_{i}^{*}$ is $p$-dominant for player $i$ at $x$, if $a_{i}^{*}$ is a best response to any belief $\lambda_{-i} \in \mathcal{M}_{-i}$ that puts at least probability $p$ on opponents profile $a_{-i}^{*}$ being played. Hence $p_{i}^{a_{i}^{*}}(x)$ may be viewed as the smallest probability such that this is true for $a_{i}^{*}$. Notice that if $a_{i}^{*}$ is a best response of player $i$ to $a_{-i}^{*}$ at $x$, then 1 is in the set over which the infimum is being taken, and in this case, $p_{i}^{a_{i}^{*}}(x)$ is well-defined. Moreover, if $a_{i}^{*}$ is a strictly dominant action for player $i$ at $x$, then $p_{i}^{a_{i}^{*}}(x)=0$.

We now present two propositions which allow us to study the notion of $p$-dominance in a parameterized environment. Proposition 6 allows us to say that if action $a_{i}^{*}$ is $p$ dominant at some $x \in \mathbb{R}$, it remains so at parameters arbitrarily close to $x$, in the sense of upper semi-continuity of $p_{i}^{a_{i}^{*}}(\cdot)$. Proposition 7 provides useful bounds for the strength of $p$-dominance.

Proposition 6. Let $\Gamma$ be a global game, $a^{*}$ a profile of actions, and $X^{\prime} \subset \mathbb{R}$.

For $i \in \mathcal{I}$, if for every $x \in X^{\prime}, a_{i}^{*}$ is the unique best response of player $i$ to $a_{-i}^{*}$ at $x$, then $p_{i}^{a_{i}^{*}}$ is upper semicontinuous on $X^{\prime}$.

Proof. Given in appendix. 
Proposition 7. Let $\Gamma$ be a monotone global game and $a^{*}$ a profile of actions.

Suppose $a_{i}^{*}$ is the unique best response of player $i$ to $a_{-i}^{*}$ at $x_{i}$.

If $x_{i} \notin \mathcal{X}_{i, a_{i}^{*}}^{v}$, then

1. There is $j \neq i$ such that $B\left(x_{i}, 2 v\right) \not \subset \mathcal{X}_{j, a_{j}^{*}}^{v}$,

2. Either $p_{i}^{a_{i}^{*}}\left(x_{i}\right) \geq \mu_{i}\left(\left\{\bar{s}_{-i}^{v}=a_{-i}^{*}\right\} \mid x_{i}, v\right)$ or $p_{i}^{a_{i}^{*}}\left(x_{i}\right) \geq \mu_{i}\left(\left\{\underline{s}_{-i}^{v}=a_{-i}^{*}\right\} \mid x_{i}, v\right)$.

Proof. Suppose that player $i \in \mathcal{I}$ has strategic substitutes at $x_{i}$. By proposition 4, $x \notin \mathcal{X}_{i, a_{i}^{*}}^{v}$ implies that either there exists some $a_{i}^{\prime}<a_{i}^{*}$ such that $\Delta \pi_{i}^{v}\left(a_{i}^{*}, a_{i}^{\prime}, \bar{s}_{-i}^{v}, x\right) \leq 0$, or there exists some $a_{i}^{\prime}>a_{i}^{*}$ such that $\Delta \pi_{i}^{v}\left(a_{i}^{*}, a_{i}^{\prime}, \underline{s}_{-i}^{v}, x\right) \leq 0$.

To prove statement (1), suppose $\forall j \neq i, B\left(x_{i}, 2 v\right) \subset \mathcal{X}_{i, a_{j}^{*}}^{v}$. Then player $i$ knows that on the entire support of her beliefs, opponents will play $a_{-i}^{*}$ at $\bar{s}_{-i}^{v}$ and at $\underline{s}_{-i}^{v}$. Therefore, for every $a_{i}^{\prime}<a_{i}^{*}$, we have

$0 \geq \Delta \pi_{i}^{v}\left(a_{i}^{*}, a_{i}^{\prime}, \bar{s}_{-i}^{v}, x_{i}\right)=\int_{\mathbb{R}^{N-1}} \Delta u_{i}\left(a_{i}^{*}, a_{i}^{\prime}, a_{-i}^{*}, x\right) d \mu_{i}\left(x_{-i} \mid x_{i}, v\right)=\Delta u_{i}\left(a_{i}^{*}, a_{i}^{\prime}, a_{-i}^{*}, x_{i}\right)>0$

where the strict inequality follows because $a_{i}^{*}$ is the unique best response of player $i$ to $a_{-i}^{*}$ at $x_{i}$, and similarly, for every $a_{i}^{\prime}>a_{i}^{*}$, we have

$0 \geq \Delta \pi_{i}^{v}\left(a_{i}^{*}, a_{i}^{\prime}, \underline{s}_{-i}^{v}, x_{i}\right)=\int_{\mathbb{R}^{N-1}} \Delta u_{i}\left(a_{i}^{*}, a_{i}^{\prime}, a_{-i}^{*}, x\right) d \mu_{i}\left(x_{-i} \mid x_{i}, v\right)=\Delta u_{i}\left(a_{i}^{*}, a_{i}^{\prime}, a_{-i}^{*}, x_{i}\right)>0$

a contradiction.

To prove statement (2), suppose there exists some $a_{i}^{\prime}<a_{i}^{*}$ such that $0 \geq \Delta \pi_{i}^{v}\left(a_{i}^{*}, a_{i}^{\prime}, \bar{s}_{-i}, x_{i}\right)$. Let $\lambda_{-i}^{\prime}$ be defined by $\lambda_{-i}^{\prime}\left(a_{-i}\right)=\mu_{i}\left(\left\{\bar{s}_{-i}=a_{-i}\right\} \mid x_{i}, v\right)$. Therefore,

$$
0 \geq \Delta \pi_{i}^{v}\left(a_{i}^{*}, a_{i}^{\prime}, \bar{s}_{-i}, x_{i}\right)=\Delta \mathcal{U}_{i}\left(a_{i}^{*}, a_{i}^{\prime}, \lambda_{-i}^{\prime}, x_{i}\right)
$$

By contrapositive of proposition 9 in the appendix, it follows that $p_{i}^{a_{i}^{*}}\left(x_{i}\right) \geq \mu_{i}\left(\left\{\bar{s}_{-i}^{v}=a_{-i}^{*}\right\} \mid\right.$ $\left.x_{i}, v\right)$, as desired. Similarly, if there exists some $a_{i}^{\prime}>a_{i}^{*}$ such that $0 \geq \Delta \pi_{i}^{v}\left(a_{i}^{*}, a_{i}^{\prime}, \underline{s}_{-i}, x_{i}\right)$, then $p_{i}^{a_{i}^{*}}\left(x_{i}\right) \geq \mu_{i}\left(\left\{\underline{s}_{-i}^{v}=a_{-i}^{*}\right\} \mid x_{i}, v\right)$. The case where player $i$ has strategic complements is proved similarly. 
We now present our first main result, which states that if a profile of actions $a^{*}$ satisfies a $p$-dominance condition on an interval that intersects a dominance region for $a^{*}$, then $a^{*}$ is the only equilibrium that survives after an arbitrarily small amount of uncertainty is added to the game. (Recall that our notion of dominance region, as discussed above, only requires dominance for $I-1$ players.)

Theorem 1. Let $\Gamma$ be a monotone global game that has a dominance region for a profile of actions $a^{*}$, and let $\hat{X}= \begin{cases}\left\{x \in X_{0} \mid p_{1}^{a_{1}^{*}}(x)+p_{2}^{a_{2}^{*}}(x)<1\right\} & \text { if } I=2 \\ \left\{x \in X_{0} \mid \forall i \in \mathcal{I}, p_{i}^{a_{i}^{*}}(x)<\frac{1}{I}\right\} & \text { if } I>2 .\end{cases}$

For every open interval $\mathcal{O} \subset \hat{X}$ such that either $\mathcal{O} \cap(-\infty, \underline{x}] \neq \emptyset$, or $\mathcal{O} \cap[\bar{x}, \infty) \neq$ $\emptyset$, (depending on which dominance region exists,) we have that $\forall x \in \mathcal{O}, \exists \hat{v}>0, \forall v \in$ $(0, \hat{v}], \forall s \in \mathcal{S}^{v}, s(x)=a^{*}$.

Proof. Suppose the game has a lower dominance region (the proof is similar for upper dominance region). That is, there is $\underline{x}$ and there is $i \in \mathcal{I}$, such that $(-\infty, \underline{x}] \subset \bigcap_{j \neq i} D_{j}^{a_{j}^{*}}$. Consider an open interval $\mathcal{O} \subset \hat{X}$ such that $\mathcal{O} \cap(-\infty, \underline{x}] \neq \emptyset$. If $\mathcal{O} \subset(-\infty, \underline{x}]$ then the statement follows immediately from proposition 5. So suppose $\mathcal{O} \not \subset(-\infty, \underline{x}]$. As $\mathcal{O}$ is an open interval, this implies that $\underline{x} \in \mathcal{O}$. As $\underline{x} \in \bigcap D_{j}^{a_{j}^{*}}$, proposition 5 implies that there exists $\bar{v}>0$ such that for all $v<\bar{v}, B(\underline{x}, 2 v) \subset \bigcap_{i \in \mathcal{I}}^{j \neq i} \mathcal{X}_{i, a_{i}^{*}}^{v}$, and we may further suppose that $\left[\underline{x}_{0}-2 v, \bar{x}_{0}+2 v\right]$ is contained in the interior of the support of $f$ (so that proposition 2 can be applied).

Suppose by way of contradiction that $\exists \hat{x} \in \mathcal{O}, \forall \hat{v}>0, \exists v \in(0, \hat{v}], \exists \hat{s} \in \mathcal{S}^{v}, \hat{s}(\hat{x}) \neq a^{*}$. In this case, $\underline{x}<\hat{x}$. Let $\overline{\bar{v}}$ be such that $B(\hat{x}, \overline{\bar{v}}) \subset \mathcal{O}$, and define the sequence $\left(v_{n}\right)_{n=0}^{\infty}$ as follows. For $n=0$, let $v_{0} \leq \min \{\bar{v}, \overline{\bar{v}}\}$ be such that $s^{0}(\hat{x}) \neq a^{*}$ for some $s^{0} \in \mathcal{S}^{v_{0}}$. For $n \geq 1$, let $v_{n} \leq \frac{v_{n-1}}{2^{n}}$ be such that $s^{n}(\hat{x}) \neq a^{*}$ for some $s^{n} \in \mathcal{S}^{v_{n}}$. Notice that $v_{n} \rightarrow 0$.

For every player $i \in \mathcal{I}$, let $x_{i}^{v_{n}}=\sup \left\{x \mid[\underline{x}, x) \subset \mathcal{X}_{i, a_{i}^{*}}^{v_{n}}\right\}$. Notice that $v_{n} \leq \bar{v}$ implies that the set over which the supremum is being taken is nonempty so that each $x_{i}^{v_{n}}$ is well defined, and each $x_{i}^{v_{n}} \notin \mathcal{X}_{i, a_{i}}^{v_{n}}$. Moreover, $s^{n}(\hat{x}) \neq a^{*}$ implies that there is some $i \in \mathcal{I}$ such that $x_{i}^{v_{n}} \leq \hat{x}<\infty$. Thus, if we define $x_{\ell}^{v_{n}}=\min _{i} x_{i}^{v_{n}}$, then for every $n, x_{\ell}^{v_{n}} \in[\underline{x}, \hat{x}]$. Also, 
let $x_{k}^{v_{n}}$ denote the second largest of the $x_{i}^{v_{n}}$. The following claims help complete the proof.

Claim 1. For every $v_{n}, x_{k}^{v_{n}} \in\left[x_{\ell}^{v_{n}}, x_{\ell}^{v_{n}}+2 v_{n}\right]$. To see this is true, suppose $x_{\ell}^{v_{n}}+2 v_{n}<$ $x_{k}^{v_{n}}$, which implies that $x_{\ell}^{v_{n}}+2 v_{n}<x_{i}^{v_{n}}$ for all $i \neq \ell$. In other words, for all $i \neq \ell$, $B\left(x_{\ell}^{v_{n}}, 2 v_{n}\right) \subset \mathcal{X}_{i, a_{i}^{*}}^{v_{n}}$. As $x_{\ell}^{v_{n}} \notin \mathcal{X}_{\ell, a_{i}^{*}}^{v_{n}}$, this contradicts proposition 7 .

Claim 2. For every $v_{n}$, and for every $i, p_{i}^{a_{i}^{*}}\left(x_{i}^{v_{n}}\right) \geq F_{i}\left(x_{-i}^{v_{n}} \mid x_{i}^{v_{n}}, v_{n}\right)$. To see this is true, notice that $x_{i}^{v_{n}} \notin \mathcal{X}_{i, a_{i}^{*}}^{v_{n}}$, and therefore, proposition 7 implies that either $p_{i}^{a_{i}^{*}}\left(x_{i}^{v_{n}}\right) \geq$ $\mu_{i}\left(\left\{\bar{s}_{-i}^{v_{n}}=a_{-i}^{*}\right\} \mid x_{i}^{v_{n}}, v_{n}\right)$ or $p_{i}^{a_{i}^{*}}\left(x_{i}^{v_{n}}\right) \geq \mu_{i}\left(\left\{\underline{s}_{-i}^{v_{n}}=a_{-i}^{*}\right\} \mid x_{i}^{v_{n}}, v_{n}\right)$. Suppose WLOG that the latter case is true. Notice that $\left\{\xi \mid \xi \ll x_{-i}^{v_{n}}\right\} \subset\left\{\xi \mid \underline{s}_{-i}^{v_{n}}(\xi)=a_{-i}^{*}\right\}$, and therefore,

$$
\begin{aligned}
p_{i}^{a_{i}^{*}}\left(x_{i}^{v_{n}}\right) & \geq \mu_{i}\left(\left\{\underline{s}_{-i}^{v_{n}}=a_{-i}^{*}\right\} \mid x_{i}^{v_{n}}, v_{n}\right) \\
& \geq \mu_{i}\left(\left\{\xi \ll x_{-i}^{v_{n}}\right\} \mid x_{i}^{v_{n}}, v_{n}\right) \\
& =\mu_{i}\left(\left\{\xi \leq x_{-i}^{v_{n}}\right\} \mid x_{i}^{v_{n}}, v_{n}\right) \\
& =F_{i}^{v_{n}}\left(x_{-i}^{v_{n}} \mid x_{i}^{v_{n}}, v_{n}\right)
\end{aligned}
$$

where the first equality follows because $\mu_{i}$ is absolutely continuous with respect to Lebesgue measure on $\mathbb{R}^{I-1}$.

Claim 3. If there are two players only, then for all $\epsilon>0$, there exists $N>0$, such that for all $n \geq N$,

$$
F_{1}\left(x_{2}^{v_{n}} \mid x_{1}^{v_{n}}, v_{n}\right)+F_{2}\left(x_{1}^{v_{n}} \mid x_{2}^{v_{n}}, v_{n}\right)=F_{\ell}\left(x_{k}^{v_{n}} \mid x_{\ell}^{v_{n}}, v_{n}\right)+F_{k}\left(x_{\ell}^{v_{n}} \mid x_{k}^{v_{n}}, v_{n}\right) \geq 1-\epsilon .
$$

This follows directly from the discussion after proposition 2 .

Claim 4. If there are more than two players, then for all $\epsilon>0$, there exists $N>0$, such that for all $n \geq N$,

$$
F_{\ell}\left(x_{\ell}^{v_{n}} \mathbf{1}_{I-1} \mid x_{\ell}^{v_{n}}, v_{n}\right) \geq \frac{1}{I}-\epsilon .
$$

This also follows from the discussion after proposition 2 .

Claims 1, 2, and 3 give us a contradiction for the case of two players, as follows. By claims 2 and 3, we have that for each $\epsilon>0, \exists N, \forall n \geq N$,

$$
p_{1}^{a_{1}^{*}}\left(x_{1}^{v_{n}}\right)+p_{2}^{a_{2}^{*}}\left(x_{2}^{v_{n}}\right)=p_{\ell}^{a_{\ell}^{*}}\left(x_{\ell}^{v_{n}}\right)+p_{k}^{a_{k}^{*}}\left(x_{k}^{v_{n}}\right) \geq 1-\epsilon .
$$


As each $x_{\ell}^{v_{n}} \in[\underline{x}, \hat{x}]$, passing to a subsequence if necessary, suppose $x_{\ell}^{v_{n}}$ converges to some $x^{*} \in[\underline{x}, \hat{x}]$. By claim 1, we then see that the sequences $\left(x_{\ell}^{v_{n}}\right)$ and $\left(x_{k}^{v_{n}}\right)$, and hence the sequences $\left(x_{1}^{v_{n}}\right)$ and $\left(x_{2}^{v_{n}}\right)$ share the common limit $x^{*}$. Using the upper semi-continuity of

$p_{i}^{a_{i}^{*}}$ and then by making $\epsilon$ arbitrarily small, we conclude that $p_{1}^{a_{1}^{*}}\left(x^{*}\right)+p_{2}^{a_{2}^{*}}\left(x^{*}\right) \geq 1$. On the other hand, $x^{*} \in[\underline{x}, \hat{x}] \subset \hat{X}$, and therefore, $p_{1}^{a_{1}^{*}}\left(x^{*}\right)+p_{2}^{a_{2}^{*}}\left(x^{*}\right)<1$, a contradiction.

Claims 2 and 4 give us a contradiction for the case of more than two players, as follows. As $x_{\ell}^{v_{n}}$ is the smallest of all $x_{i}^{v_{n}}$, it follows that $F_{\ell}\left(x_{-\ell}^{v_{n}} \mid x_{\ell}^{v_{n}}, v^{n}\right) \geq F_{\ell}\left(x_{\ell}^{v_{n}} \mathbf{1}_{I-1} \mid x_{\ell}^{v_{n}}, v^{n}\right)$. Therefore, by claims 2 and 4 , we have that for each $\epsilon>0, \exists N, \forall n \geq N$,

$$
p_{\ell}^{a_{\ell}^{*}}\left(x_{\ell}^{v_{n}}\right) \geq \frac{1}{I}-\epsilon
$$

As earlier, suppose $x_{\ell}^{v_{n}} \rightarrow x^{*} \in[\underline{x}, \hat{x}]$ and going to a subsequence and relabeling if necessary, suppose $\ell$ is constant along the sequence. Using upper semi-continuity of $p_{\ell}^{a_{\ell}^{*}}$, and then by making $\epsilon$ arbitrarily small, we conclude that

$$
p_{\ell}^{a_{\ell}^{*}}\left(x_{\ell}^{*}\right) \geq \frac{1}{I}
$$

On the other hand, $x^{*} \in[\underline{x}, \hat{x}] \subset \hat{X}$ implies $p_{\ell}^{a_{\ell}^{*}}\left(x^{*}\right)<\frac{1}{I}$, a contradiction.

\section{$3 \quad$ Examples}

Example 1 (Deterrence). Consider a scenario of deterrence between two countries modeled by a game of Chicken. Each country must decide between an aggressive strategy (A) or capitulation (C). We follow the formulation of Baliga and Sjostrom (2012) by allowing $h_{i}$ to be Player $i$ 's preference for aggression, $c$ and $d$ to be the respective costs of being aggressive or capitulating in the face of an aggressive opponent, and normalize the payoffs of mutual capitulation to 0. By assuming that $0<h_{i}<c-d$ for each player, we have a game of Chicken (GSS) with two strict Nash equilibrium $(A, C)$ and $(C, A)$, represented below: 


\section{$P 2$}

\begin{tabular}{cc|c|c|}
\multicolumn{4}{c}{$A$} \\
\multicolumn{1}{c}{$A$} \\
\cline { 3 - 3 }$P 1$ & $A$ & $h_{1}-c, h_{2}-c$ & $h_{1},-d$ \\
\cline { 3 - 4 } & $C$ & $-d, h_{2}$ & 0,0 \\
\cline { 3 - 4 } & & &
\end{tabular}

Examples of such situations where capitulating to an attacking opponent are numerous. In these types of examples, only one of the two equilibria emerges, with one party capitulating to the other. For example, Kilgour and Zagare (1991) formulate a dynamic game in which each player is uncertain about the opponent's preferences and conclude that a player will capitulate if they perceive a high enough probability of aggression from the other party, which they deem a "credible threat".

Alternatively, suppose that there is some amount of uncertainty concerning player 1's preference for aggression, which we model by allowing $h_{1}=x$. Then for $0<x \leq c-d$ we have multiple equilibria as before, but for $x>c-d$ we have that $A$ is strictly preferred for Player 1. Also, $(A, C)$ is a strict Nash equilibrium for all $x>0$. Calculating $p_{1}(x)$ and $p_{2}(x)$ gives $p_{2}(x)=\frac{h_{2}}{c-d}$ and

$$
p_{1}(x)=\left\{\begin{array}{rl}
\frac{c-d-x}{c-d} & 0<x<c-d \\
0 & x \geq c-d
\end{array}\right.
$$

Then, we find that the condition

$$
p_{1}(x)+p_{2}(x)<1
$$

is satisfied for all $x>h_{2}$. This suggests that player 2 will capitulate as long as she observes a preference for aggression from player 1 that is at least slightly higher than her own, and there is a small amount of uncertainty in this observation.

Note that this is an example of a GSS with only one dominance region, and that this dominance region is only for one of the two players (player 1). These characteristics imply that the methods of Frankel, Morris, and Pauzner (2003) and Harrison and Jara-Moroni (2016) cannot be applied. 
Example 2 (Technology Adoption). Consider the following, slightly modified 3-player version of the technology adoption model considered in Keser, Suleymanova, and Wey (2012). It is a game with strategic complements (GSC). Three players must simultaneously decide whether to adopt an inferior technology $A$ or a superior technology $B$. The benefit to each player $i \in\{1,2,3\}$ of adopting a specific technology $t \in\{A, B\}$ is given by

$$
U_{t}\left(N_{t}\right)=v_{t}+\gamma_{t}\left(N_{t}-1\right)
$$

where $N_{t}$ is the total number of players using technology $t, v_{t}>0$ is the stand-alone benefit from using technology $t$, and $\gamma_{t}>0$ is the benefit derived from the network effect of adopting the technology of others. Suppose technology $B$ is superior to technology A, formalized by $v_{B}>v_{A}$. The stand-alone benefit of technology $A$ is normalized to 1 (that is, $v_{A}=1$ ) and suppose the network effect benefit of technology $B$ is greater than that of technology $A$ (in particular, suppose $\gamma_{A}=1$ and $\gamma_{B}=3$ ). ${ }^{6}$ Suppose the stand-alone benefit from technology $B$ is uncertain and can vary, captured by setting $v_{B}=x$. Payoffs are as follows.

$$
\begin{aligned}
& \begin{array}{lll}
A & P 3 & B
\end{array} \\
& P 2 \quad P 2
\end{aligned}
$$

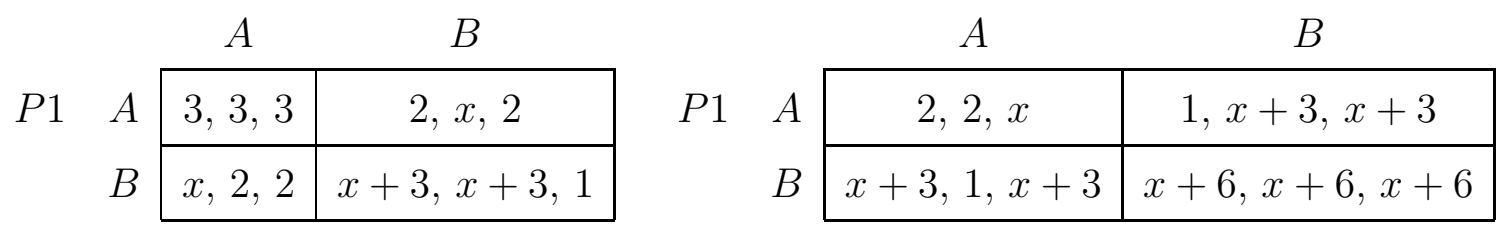

Notice that for $x \in[1,3]$, both $(A, A, A)$ and $(B, B, B)$ are strict Nash equilibria, and for $x>3,(B, B, B)$ is the unique strictly dominant action profile, giving an "upper dominance region". However, as technology $B$ is better than $A$, there is a natural lower bound on $x>v_{A}=1$, and at the lower bound there are multiple equilibria. Thus, there is no "lower dominance region". ${ }^{7}$ In particular, the framework in FMP cannot be applied.

Nevertheless, this example fits naturally in our framework. In order to apply theorem

\footnotetext{
${ }^{6}$ This can be generalized easily.

${ }^{7}$ Nothing really changes if we allow the marginal benefit of technology $B$ over $A$ to be bounded below.
} 
1, we have that for each $i \in\{1,2,3\}$,

$$
p_{i}^{a_{i}^{*}}(x)=\left\{\begin{array}{cl}
\frac{3-x}{8} & 1<x<3 \\
0 & x \geq 3
\end{array} .\right.
$$

As $x>1$, it follows that $p_{i}^{a_{i}^{*}}(x)<\frac{1}{3}$ for all $i \in I$, and therefore, $(B, B, B)$ is the unique global games prediction for every $x \in[1,3]$. In this example, indeterminacy is completely resolved.

As an aside, notice that an ancillary technical benefit of requiring only one dominance region is that it may help us sidestep some results such as Weinstein and Yildiz (2007) and Basteck, Daniëls, and Heinemann (2013). ${ }^{8}$ The richness condition in Weinstein and Yildiz (2007) would require two dominance regions here, and a re-parameterization of the example á la Basteck, Daniëls, and Heinemann (2013) would automatically satisfy the richness condition of Weinstein and Yildiz (2007), thereby limiting the application of global games in this case. In other words, a given situation may have natural parameterizations that fall outside the scope of these papers. In that case, if the conditions here are satisfied, the global games method remains applicable.

Example 3 (Combining strategic complements and substitutes). The framework of monotone global games allows for combination of strategic complements and strategic substitutes in the same formulation.

Suppose there are two types of investment choices. In the first type, the choice is between a sure benchmark payoff, normalized to 0 , or a linear uncertain payoff, $x \in \mathbb{R}$. In the second type, the choice is between a sure benchmark payoff, normalized to 1 , or a quadratic payoff given by squared deviations from the benchmark, $(x-1)^{2}$, for $x \in \mathbb{R}{ }^{9}$

\footnotetext{
${ }^{8}$ Basteck, Daniëls, and Heinemann (2013) uses two dominance regions and state monotonicity assumptions common in the literature.

${ }^{9}$ Intuitively, large deviations from the benchmark may be profitable, because there is no cost to achieve the benchmark payoff and we may invest in costly technology to profit from larger deviations from the norm, whether it is more sophisticated short sale type technology to benefit from large negative shocks
} 
Consider the following two player game. Each player may take one of two actions: $L$ or $H$, with $L \prec H$, and payoffs are parameterized by $x \in \mathbb{R}$.

\section{$P 2$}

\begin{tabular}{|c|c|c|}
\hline & $L$ & $H$ \\
\hline$P 1 \quad L$ & $0,(x-1)^{2}$ & 1,1 \\
\hline$H$ & $x, x$ & $(x-1)^{2}, 0$ \\
\hline
\end{tabular}

Player 1 payoffs are motivated as follows. If $\mathrm{P} 2$ plays $L, \mathrm{P} 1$ payoff is given by the first type of investment choice; if she plays $L$, she gets 0 and if she plays $H$, she gets $x$. If P2 plays $H, \mathrm{P} 1$ payoff is given by the second type of investment choice; if she plays $L$, she gets 1 and if she plays $H$, she gets $(x-1)^{2}$. Player 2 payoffs are motivated similarly.

This formulation allows for both GSC and GSS depending on the parameter $x$. For $x<0$, this is a GSC with two strict Nash equilibria $(L, L)$ and $(H, H)$, and for $x \in(0,2)$, it is a GSS with two strict Nash equilibria $(H, L)$ and $(L, H) \cdot{ }^{10}$ Moreover, for $x>2$, the game has a unique strictly dominant equilibrium $(H, L)$. Notice that there is only one dominance region in this example.

We investigate the global games selection by calculating $p_{1}^{H}(x)$ and $p_{2}^{L}(x)$, given by

$$
p_{1}^{H}(x)=p_{2}^{L}(x)=\left\{\begin{array}{cl}
\frac{1-(x-1)^{2}}{1-(x-1)^{2}+x} & 0<x<2 \\
0 & x \geq 2
\end{array}\right.
$$

For $x \in(0,2), p_{1}(x)+p_{2}(x)<1$ is satisfied for all $x>1$. Therefore, multiplicity is resolved on $(1,2]$.

or better fundamental research to benefit from large positive shocks. These scale type economies may not be profitable for small deviations from the norm.

${ }^{10}$ One may consider reversing the order on the strategy space of one player to transform a two player GSS into a GSC. That would just change the role of GSS and GSC in this example. That type of order reversal does not work for a three player version with strategic heterogeneity given below. As an aside, we work with the standard assumption that the complete information game has a given order on action spaces and that order is fixed as part of the definition of the global game. Our results apply without any need to change orders. 
This example can be extended to a three player game with strategic heterogeneity, as shown in the next section.

\section{Example 4 (Market Entry).}

Consider the following three firm example in the framework of Brander and Spencer (1985). In this game, players 1 and 2 are domestic firms, and player 3 is a potential foreign competitor. Each firm must decide to either enter $(E)$ or stay out $(S)$ of the market. Payoffs are given as follows.

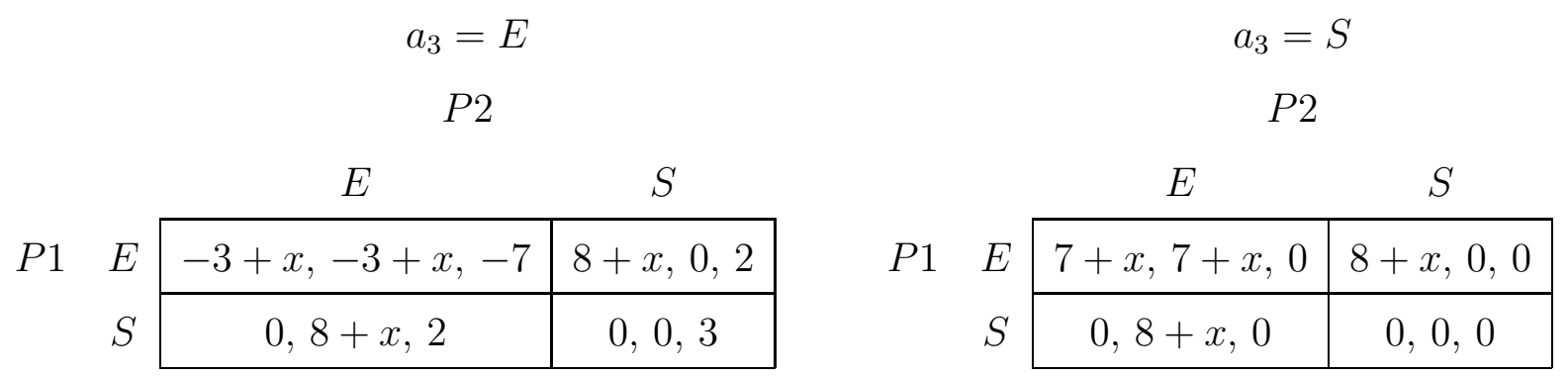

Intuitively, the domestic market can only support two firms making a positive profit. The foreign firm has higher costs for producing in the domestic market. The more competitors in a market, the less profit is earned by each firm. In this game, the domestic firms may receive a subsidy $x \geq 0$ in order to make them more competitive, similar to a stylized version of Brander and Spencer (1985). Notice that for every $x \geq 0$, this is a GSS. $^{11}$ Moreover, for $x \in[0,3],(S, E, E),(E, S, E)$, and $(E, E, S)$ are equilibria, while for $x>3,(E, E, S)$ is the unique Nash equilibrium with $E$ being a dominant strategy for player 1 and 2, while player 3 has no dominant strategy. Thus, there is only one dominance region (for two of the three players). These features rule out application of existing results in the literature.

We will determine when $(E, E, S)$ is the global games selection. Calculating $p(x)$ for each player for this strategy, we get

$$
p_{i}(x)=\frac{3-x}{10}
$$

\footnotetext{
${ }^{11}$ This is a three player GSS and we can not flip orders in order to make this a GSC.
} 
for player $i=1,2$, and hence this is less than $\frac{1}{3}$ for all $x>-\frac{1}{3}$, so that this is true on the entire space of uncertainty $[0, \infty)$. Moreover, we have that

$$
p_{3}(x)=\frac{3}{10}
$$

which is less than $\frac{1}{3}$ for all $x$. Once again, this is true on the entire space of uncertainty $[0, \infty)$. Thus, indeterminacy is eliminated completely in this model. An interpretation is that even a "threat" of a subsidy may be enough to drive foreign competition out of the market.

\section{Group Partitions}

Notice that the $p$-dominance condition in theorem 1

$$
p_{i}^{a_{i}^{*}}(x)<\frac{1}{I}
$$

becomes more restrictive as the number of players $I$ increases. This is the direct result of the requirement that each player must keep track of all possible actions her opponents may play, which depends on the signals that each may receive. As I becomes larger, the computations related to player $i$ 's beliefs over the signals of opponents make the p-dominance condition for each player more demanding.

One way in which this condition may be relaxed is by assuming that each player believes that a particular group of her opponents receives the same signal. In this case, the belief over the signal of each player in this group is the same. This does not restrict payoffs for players in a group, and allows the same level of heterogeneity in player payoffs as considered earlier, but considering players in (a small number of) groups that receive the same signal can relax the $p$-dominance condition, as shown in more detail below.

One motivation for this approach is that in some cases groups of agents may naturally observe the same signal of the underlying state of the world. We model this phenomenon 
by assuming that there are groups of players, and each player in a given group observes the same signal as others in the same group. We term this the case of fixed group partitions.

Another motivation is the idea of cognitive shortcuts from social psychology. In other words, keeping track of beliefs for every opponent is a cognitively daunting task when the number of opponents is large. In this case, decision makers may employ a cognitive shortcut by exhibiting "stereotyping behavior", which leads them to infer more correlation about opponents' types than would be present if they were to correctly form beliefs for each player separately. As Macrae and Bodenhausen (2000) write, "Given basic cognitive limitations and a challenging stimulus world, perceivers need some way to simplify and structure the person perception process." This, they write, leads agents to emphasize "stereotype-consistent information" to a greater extent.

We model this phenomenon by assuming that player $i$ believes that there are groups of opponents, and players in the same group receive the same signal. Instances of this type have been previously implemented in the economics literature. Most notably, Healy (2007) studies reputation building equilibria in repeated labor market games by assuming that firms stereotype workers, so that firms believe that all workers are of the same type, or highly correlated with one another, regardless of the fact that this may not represent the true type distrubtion. We term this the case of subjective group partitions.

By accounting for such tendencies, theorems 2 and 3 below show that theorem 1 may be modified so that the $I$ used in equation 4 is replaced with the number of groups. For example, when players group all other opponents into one group of "stereotypes", then even when $I$ is large, the requirements of theorem 1 are no more restrictive than in the case of 2 players.

\subsection{Fixed Group Partitions}

Definition 2. We say that $\mathcal{G}=\left\{G_{1}, G_{2}, \ldots, G_{K}\right\}$ is a group partition of $\mathcal{I}$, if 
1. $\mathcal{G}$ is a finite partition of $\mathcal{I}$, that is, a collection of nonempty, disjoint sets whose union is $\mathcal{I}$, and $K \geq 2$ is the finite number of groups in $\mathcal{G}$.

2. Each player $i \in \mathcal{I}$ forms her beliefs as in definition 1 under the assumption that in each group, every player in the group receives the same signal. That is, for every player $i$,

$$
x_{i}=x+v \epsilon_{G_{k(i)}},
$$

where $k(i)$ is the unique index $k$ identifying the group $G_{k}$ to which $i$ belongs; that is, $i \in G_{k(i)}$. Moreover, as earlier, $\left\{\epsilon_{G_{k}}\right\}_{k=1, \ldots, K}$ are i.i.d., jointly independent with $f$, and drawn from $[-1,1]$ according to atomless, continuous, and symmetric density $\phi_{k}$. When convenient, we may denote the group to which $i$ belongs as $G(i)$ and write the realizations for that group as $x_{G}$ or $x_{G(i)}$.

Conditional on group $G$ receiving signal $x_{G}$, we denote the conditional distribution of signals received by other groups as $\mu_{G}\left(x_{-G} \mid x_{G}\right)$. For notational convenience, if player $i$ receives signal $x_{i}$, we denote this conditional distribution by $\mu_{i}\left(x_{-G(i)} \mid x_{i}\right)$.

One feature of this formulation is that if player $i$ observes $x_{i}$, then she knows for sure what every opponent in her group observes. Therefore, for opponents in the same group as player $i$, there is no uncertainty, and the global games method is not applicable to these in-group opponents. The analysis needs to be modified to accommodate this feature, as described below.

Having observed signal $x_{i}$, the expected payoff to player $i$ from playing action $a_{i} \in A_{i}$ against a profile of opponents strategies $s_{-i} \in \times_{j \neq i} \mathcal{S}_{j}$ is given by

$$
\pi_{i}^{v}\left(a_{i}, s_{-i}, x_{i}\right)=\int_{\mathbb{R}^{K-1}} u_{i}\left(a_{i}, s_{G(i)}\left(x_{i}\right), s_{-G(i)}\left(x_{-G(i)}\right), x_{i}\right) d \mu_{i}\left(x_{-G(i)} \mid x_{i}, v\right),
$$

where $s_{G(i)}\left(x_{i}\right)=\left(s_{j}\left(x_{i}\right)\right)_{j \in G(i), j \neq i}$ are the actions played by in-group opponents of player $i$, and $s_{-G(i)}\left(x_{-G(i)}\right)=\left(s_{j}\left(x_{j}\right)_{j \notin G(i)}\right.$ are the actions played by outside-group opponents of player $i$. This incorporates the feature that if player $i$ observes signal $x_{i}$, then she knows for sure that other players in her group also observe $x_{i}$. The (expected) marginal 
benefit to player $i$ of playing action $\hat{a}_{i}$ over $\tilde{a}_{i}$ at $x_{i}$ when opponents are playing $s_{-i}$ is $\Delta \pi_{i}^{v}\left(\hat{a}_{i}, \tilde{a}_{i}, s_{-i}, x_{i}\right)=\pi_{i}^{v}\left(\hat{a}_{i}, s_{-i}, x_{i}\right)-\pi_{i}^{v}\left(\tilde{a}_{i}, s_{-i}, x_{i}\right)$. With these definitions, the iterative procedure remains the same.

Moving toward the notion of $p$-dominance in this case, the notion of expected utility over opponents outside a given player's group is defined as follows. Let $\mathcal{M}_{-G(i)}$ denote the set of probability distributions over the actions of out-group opponents of $i\left(\times_{j \notin G(i)} A_{j}\right)$. Expected payoff to player $i$ from playing $a_{i}$ at $x_{i}$ when in-group opponents are playing $\left(a_{j}\right)$ and out-group opponents are playing distribution $\lambda_{-G(i)} \in \mathcal{M}_{-G(i)}$ is

$$
\mathcal{U}_{i}\left(a_{i},\left(a_{j}\right), \lambda_{-i}, x_{i}\right)=\Sigma_{a_{-G(i)}} \lambda_{-G(i)}\left(a_{-G(i)}\right) u_{i}\left(a_{i},\left(a_{j}\right), a_{-G(i)}, x_{i}\right)
$$

and the expected marginal benefit of $\hat{a}_{i}$ over $\tilde{a}_{i}$ is

$$
\Delta \mathcal{U}_{i}\left(\hat{a}_{i}, \tilde{a}_{i},\left(a_{j}\right), \lambda_{-G(i)}, x_{i}\right)=\mathcal{U}_{i}\left(\hat{a}_{i},\left(a_{j}\right), \lambda_{-G(i)}, x_{i}\right)-\mathcal{U}_{i}\left(\tilde{a}_{i},\left(a_{j}\right), \lambda_{-G(i)}, x_{i}\right) .
$$

As earlier, given a profile of strategies for opponents of player $i, s_{-i}^{\prime} \in \mathcal{S}_{-i}$, define $\lambda_{-G(i)}^{\prime} \in \mathcal{M}_{-G(i)}$ as $\lambda_{-G(i)}^{\prime}\left(a_{-G(i)}\right)=\mu_{i}\left(\left\{s_{-G(i)}^{\prime}=a_{-G(i)}\right\} \mid x_{i}, v\right)$. In other words, $\lambda_{-G(i)}^{\prime}\left(a_{-G(i)}\right)$ is the probability that out-group opponents of $i$ play $a_{-G(i)}$ under $s_{-G(i)}^{\prime}$. As earlier, it follows that

$$
\Delta \pi_{i}^{v}\left(a_{i}, a_{i}^{\prime}, s_{-i}^{\prime}, x_{i}\right)=\Delta \mathcal{U}_{i}\left(a_{i}, a_{i}^{\prime},\left(s_{j}^{\prime}\left(x_{i}\right)\right), \lambda_{-G(i)}^{\prime}, x_{i}\right)
$$

The notion of $p$-value is defined as follows. Let $a^{*} \in A$ be a profile of actions. For each player $i$ and for each $x \in \mathbb{R}$, let

$$
\begin{aligned}
p_{i}^{a_{i}^{*}}(x)=\inf \{\xi \in[0,1] \mid & \forall \lambda_{-G(i)} \in \mathcal{M}_{-G(i)}, \lambda_{-G(i)}\left(a_{-G(i)}^{*}\right) \geq \xi \Rightarrow \\
& \left.\forall a_{i} \neq a_{i}^{*}, \forall\left(a_{j}\right), \Delta \mathcal{U}_{i}\left(a_{i}^{*}, a_{i},\left(a_{j}\right), \lambda_{-G(i)}, x\right) \geq 0\right\} .
\end{aligned}
$$

Notice that the definition uses only probabilities on the actions of out-group opponents of player $i$. This reflects the fact that at observation $x$ for player $i$, there is no uncertainty about the realizations of in-group opponents of $i$. Moreover, we want to allow for heterogeneity in preferences of players within a group. This is reflected in the quantifier $\forall\left(a_{j}\right)$, 
where $\left(a_{j}\right)$ is shorthand for $\left(a_{j}\right)_{j \in G(i), j \neq i}$. With these defintions, theorem 1 is reformulated for this case as follows.

Theorem 2. Let $\Gamma$ be a monotone global game that has a dominance region for a profile of actions $a^{*}$, and let $\hat{X}= \begin{cases}\left\{x \in X_{0} \mid \forall i \in G_{1}, \forall j \in G_{2}, p_{i}^{a_{i}^{*}}(x)+p_{j}^{a_{j}^{*}}(x)<1\right\} & \text { if } K=2 \\ \left\{x \in X_{0} \mid \forall i \in \mathcal{I}, p_{i}^{a_{i}^{*}}(x)<\frac{1}{K}\right\} & \text { if } K>2 .\end{cases}$

For every open interval $\mathcal{O} \subset \hat{X}$ such that either $\mathcal{O} \cap(-\infty, \underline{x}] \neq \emptyset$, or $\mathcal{O} \cap[\bar{x}, \infty) \neq$ $\emptyset$, (depending on which dominance region exists,) we have that $\forall x \in \mathcal{O}, \exists \hat{v}>0, \forall v \in$ $(0, \hat{v}], \forall s \in \mathcal{S}^{v}, s(x)=a^{*}$.

Proof. Follow the proof of theorem 1 until definition of $x_{i}^{v_{n}}$. That is, for every player $i \in \mathcal{I}$, let $x_{i}^{v_{n}}=\sup \left\{x \mid[\underline{x}, x) \subset \mathcal{X}_{i, a_{i}^{*}}^{v_{n}}\right\}$. Let $x_{\ell}^{v_{n}}=\min _{i} x_{i}^{v_{n}}$ and let $x_{k}^{v_{n}}$ denote the smallest among the out-group opponents of $\ell$, that is, $x_{k}^{v_{n}}=\min _{i \notin G(\ell)} x_{i}^{v_{n}}$.

Claim 1'. For every $v_{n}, x_{k}^{v_{n}} \in\left[x_{\ell}^{v_{n}}, x_{\ell}^{v_{n}}+4 v_{n}\right]$. To see this is true, suppose $x_{\ell}^{v_{n}}+4 v_{n}<x_{k}^{v_{n}}$, which implies that $x_{\ell}^{v_{n}}+4 v_{n}<x_{i}^{v_{n}}$ for all $i \notin G(\ell)$. In other words, for all $i \notin G(\ell)$, $B\left(x_{\ell}^{v_{n}}, 4 v_{n}\right) \subset \mathcal{X}_{i, a_{i}^{*}}^{v_{n}}$. In this case, player $\ell$ knows that on the entire support of her beliefs $B\left(x_{\ell}^{v_{n}}, 2 v_{n}\right)$, out-group opponents will play $a_{-G(\ell)}^{*}$ at every $s_{-G(\ell)} \in\left[\underline{s}_{-G(\ell)}, \bar{s}_{-G(\ell)}\right]$.

Let $\lambda_{-G(\ell)}$ put a full probability atom on $a_{-G(\ell)}^{*}$. Then $\lambda_{-G(\ell)}\left(a_{-G(\ell)}^{*}\right)=1>p_{\ell}^{a_{\ell}^{*}}\left(x_{\ell}^{v_{n}}\right)$, and therefore, for every $s_{-\ell} \in\left[\underline{s}^{v_{n}}, \bar{s}^{v_{n}}\right]$ and for every $a_{\ell}^{\prime} \neq a_{\ell}^{*}$,

$$
\begin{aligned}
\Delta \pi_{\ell}^{v_{n}}\left(a_{\ell}^{*}, a_{\ell}^{\prime},\left(s_{j}\left(x_{\ell}^{v_{n}}\right)\right), s_{-G(\ell)}, x_{\ell}^{v_{n}}\right) & =\Delta \mathcal{U}_{\ell}\left(a_{\ell}^{*}, a_{\ell}^{\prime},\left(s_{j}\left(x_{\ell}^{v_{n}}\right)\right), \lambda_{-G(\ell)}, x_{\ell}^{v_{n}}\right) \\
& \geq \min _{j \in G(\ell), j \neq \ell} \Delta \mathcal{U}_{\ell}\left(a_{\ell}^{*}, a_{\ell}^{\prime},\left(a_{j}\right), \lambda_{-G(\ell)}, x_{\ell}^{v_{n}}\right) \\
& >0
\end{aligned}
$$

where the strict inequality follows from definition of $p_{\ell}^{a_{\ell}^{*}}\left(x_{\ell}^{v_{n}}\right)$. It follows that for every $s_{-\ell} \in\left[\underline{s}^{v_{n}}, \bar{s}^{v_{n}}\right]$ and for every $a_{\ell}^{\prime} \neq a_{\ell}^{*}$,

$$
\Delta \pi_{\ell}^{v_{n}}\left(a_{\ell}^{*}, a_{\ell}^{\prime}, s_{-\ell}, x_{\ell}^{v_{n}}\right) \geq \min _{j \in G(\ell), j \neq \ell} \Delta \pi_{\ell}^{v_{n}}\left(a_{\ell}^{*}, a_{\ell}^{\prime},\left(a_{j}\right), a_{-G(\ell)}^{*}, x_{\ell}^{v_{n}}\right)>0 .
$$

As the expression on the right is continuous in $x_{\ell}$, it follows that there is $\epsilon$ such that for every $x_{\ell} \in B\left(x_{\ell}^{v_{n}}, \epsilon\right)$, for every $a_{\ell}^{\prime} \neq a_{\ell}^{*}$, and for every $s_{-\ell} \in\left[\underline{s}^{v_{n}}, \bar{s}^{v_{n}}\right], \Delta \pi_{\ell}^{v_{n}}\left(a_{\ell}^{*}, a_{\ell}^{\prime}, s_{-\ell}, x_{\ell}\right)>$ 0 , whence $x_{\ell} \in \mathcal{X}_{\ell, a_{\ell}^{*}}^{v_{n}}$. This contradicts the definition of $x_{\ell}^{v_{n}}$. 
Claim 2'. For every $v_{n}$ and for every player $i$, if player $i$ has strategic substitutes at $x_{i}^{v_{n}}$ and does not have strategic complements at $x_{i}^{v_{n}}$, then either $p_{i}^{a_{i}^{*}}\left(x_{i}^{v_{n}}\right) \geq \mu_{i}\left(\left\{\bar{s}_{-G(i)}^{v_{n}}=a_{-G(i)}^{*}\right\} \mid\right.$ $\left.x_{i}^{v_{n}}, v_{n}\right)$ or $p_{i}^{a_{i}^{*}}\left(x_{i}^{v_{n}}\right) \geq \mu_{i}\left(\left\{\underline{s}_{-G(i)}^{v_{n}}=a_{-G(i)}^{*}\right\} \mid x_{i}^{v_{n}}, v_{n}\right)$.

$$
\text { Suppose } p_{i}^{a_{i}^{*}}\left(x_{i}^{v_{n}}\right)<\mu_{i}\left(\left\{\bar{s}_{-G(i)}^{v_{n}}=a_{-G(i)}^{*}\right\} \mid x_{i}^{v_{n}}, v_{n}\right) \text { and } p_{i}^{a_{i}^{*}}\left(x_{i}^{v_{n}}\right)<\mu_{i}\left(\left\{\underline{s}_{-G(i)}^{v_{n}}=a_{-G(i)}^{*}\right\} \mid\right.
$$
$\left.x_{i}^{v_{n}}, v_{n}\right)$. This implies that for $a_{i}<a_{i}^{*}$,

$$
0<\Delta \pi_{i}^{v_{n}}\left(a_{i}^{*}, a_{i}^{\prime}, \bar{a}_{G(i)}, \bar{s}_{-G(i)}^{v_{n}}, x_{i}^{v_{n}}\right) \leq \Delta \pi_{i}^{v_{n}}\left(a_{i}, a_{i}^{\prime}, \bar{s}_{-i}^{v_{n}}, x_{i}^{v_{n}}\right)
$$

where $\bar{a}_{G(i)}=\left(\bar{a}_{j}\right)_{j \in G(i), j \neq i}$. Here, the first inequality follows from definition of $p_{i}^{a_{i}^{*}}\left(x_{i}^{v_{n}}\right)$ and $p_{i}^{a_{i}^{*}}\left(x_{i}^{v_{n}}\right)<\mu_{i}\left(\left\{\bar{s}_{-G(i)}^{v_{n}}=a_{-G(i)}^{*}\right\} \mid x_{i}^{v_{n}}, v_{n}\right)$, and the second inequality follows from strategic substitutes at $x_{i}^{v_{n}}$. Moreover, the term $\Delta \pi_{i}^{v_{n}}\left(a_{i}^{*}, a_{i}^{\prime}, \bar{a}_{G(i)}, \bar{s}_{-G(i)}^{v_{n}}, x_{i}^{v_{n}}\right)$ is continuous in $x_{i}$, because in-group opponent actions do not depend on $x_{i}$ in this expression. Therefore, there is $\epsilon>0$ such that for every $\epsilon^{\prime} \in(0, \epsilon)$, player $i$ has strategic substitutes at $x_{i}^{v_{n}}+\epsilon^{\prime}{ }^{12}$ and

$$
0<\Delta \pi_{i}^{v_{n}}\left(a_{i}^{*}, a_{i}^{\prime}, \bar{a}_{G(i)}, \bar{s}_{-G(i)}^{v_{n}}, x_{i}^{v_{n}}+\epsilon\right) \leq \Delta \pi_{i}^{v_{n}}\left(a_{i}, a_{i}^{\prime}, \bar{s}_{-i}^{v_{n}}, x_{i}^{v_{n}}+\epsilon\right)
$$

where the second inequality follows from strategic substitutes. Similarly, we may choose $\epsilon$ to also satisfy that for every $\epsilon^{\prime} \in(0, \epsilon)$ and for every $a_{i}<a_{i}^{*}, \Delta \pi_{i}^{v_{n}}\left(a_{i}, a_{i}^{\prime}, \underline{s}_{-i}^{v_{n}}, x_{i}^{v_{n}}+\epsilon^{\prime}\right)>0$, for $\epsilon^{\prime} \in(0, \epsilon)$. This contradicts the definition of $x_{i}^{v_{n}}$.

An analogous statement holds if player $i$ has strategic complements at $x_{i}^{v_{n}}$ and does not have strategic substitutes at $x_{i}^{v_{n}}$. The case where player $i$ has both strategic substitutes and strategic complements at $x_{i}^{v_{n}}$ is a combination of the two cases and proved similarly.

For the final part of claim 2', using the same logic as in the last part of the proof of claim 2 in theorem 1 , it follows that for every $v_{n}$, and for every $i, p_{i}^{a_{i}^{*}}\left(x_{i}^{v_{n}}\right) \geq F_{i}\left(x_{-G(i)}^{v_{n}} \mid x_{i}^{v_{n}}, v_{n}\right)$.

Claims 3' and 4' below now follow immediately from an appropriate modification of the discussion after proposition 2 .

Claim 3'. If there are two groups only, then for all $\epsilon>0$, there is $N>0$ such that for

\footnotetext{
${ }^{12}$ Otherwise, there is a sequence $\left(x_{i}^{m}\right)$ such that player $i$ has strategic complements at $x_{i}^{m}, x_{i}^{m}>x_{i}^{v_{n}}$, and $x_{i}^{m} \rightarrow x_{i}^{v_{n}}$, implying that player $i$ has strategic complements at $x_{i}^{v_{n}}$, a contradiction.
} 
all $n \geq N$,

$$
F_{\ell}\left(x_{k}^{v_{n}} \mid x_{\ell}^{v_{n}}, v_{n}\right)+F_{k}\left(x_{\ell}^{v_{n}} \mid x_{k}^{v_{n}}, v_{n}\right) \geq 1-\epsilon
$$

Claim 4'. If there are more than two groups, then for all $\epsilon>0$, there exists $N>0$, such that for all $n \geq N, F_{\ell}\left(x_{\ell}^{v_{n}} \mathbf{1}_{K-1} \mid x_{\ell}^{v_{n}}, v_{n}\right) \geq \frac{1}{K}-\epsilon$.

For the final steps, in the case of two groups, a contradiction emerges as follows. By claims 2' and 3', we have that for each $\epsilon>0, \exists N, \forall n \geq N, p_{\ell}^{a_{\ell}^{*}}\left(x_{\ell}^{v_{n}}\right)+p_{k}^{a_{k}^{*}}\left(x_{k}^{v_{n}}\right) \geq 1-\epsilon$. As each $x_{\ell}^{v_{n}} \in[\underline{x}, \hat{x}]$, passing to a subsequence if necessary, suppose $x_{\ell}^{v_{n}}$ converges to some $x^{*} \in[\underline{x}, \hat{x}]$, and the lowest values $x_{\ell}^{v_{n}}$ are constant at a fixed $\ell$, say, in group 1 , and the second-lowest values $x_{k}^{v_{n}}$ are constant at a fixed $k$, say, in group 2 . The remainder of the proof from theorem 1 applies to get a contradiction.

For more than two groups, a contradiction emerges as follows. As $x_{\ell}^{v_{n}}$ is the smallest of all $x_{i}^{v_{n}}$, it follows that $F_{\ell}\left(x_{-G(\ell)}^{v_{n}} \mid x_{\ell}^{v_{n}}, v^{n}\right) \geq F_{\ell}\left(x_{\ell}^{v_{n}} \mathbf{1}_{K-1} \mid x_{\ell}^{v_{n}}, v^{n}\right)$.

Therefore, by claims 2 and 4, we have that for each $\epsilon>0, \exists N, \forall n \geq N$,

$$
p_{\ell}^{a_{\ell}^{*}}\left(x_{\ell}^{v_{n}}\right) \geq \frac{1}{K}-\epsilon
$$

As earlier, suppose $x_{\ell}^{v_{n}} \rightarrow x^{*} \in[\underline{x}, \hat{x}]$ and going to a subsequence and relabeling if necessary, suppose $\ell$ is constant along the sequence. Using upper semi-continuity of $p_{\ell}^{a_{\ell}^{*}}$, and then by making $\epsilon$ arbitrarily small, we conclude that

$$
p_{\ell}^{a_{\ell}^{*}}\left(x_{\ell}^{*}\right) \geq \frac{1}{K}
$$

On the other hand, $x^{*} \in[\underline{x}, \hat{x}] \subset \hat{X}$ implies $p_{\ell}^{a_{\ell}^{*}}\left(x^{*}\right)<\frac{1}{K}$, a contradiction.

\subsection{Subjective Group Partitions}

Definition 3. We say that $\mathcal{G}=\left\{\mathcal{G}_{1}, \mathcal{G}_{2}, \ldots, \mathcal{G}_{I}\right\}$ is a group partition system of $\mathcal{I}$ if the following hold: 
1. For each $i \in \mathcal{I}, \mathcal{G}_{i}$ is a group partition for player $i$, defined as follows: $\mathcal{G}_{i}=$ $\left\{G_{i, 1}, \ldots, G_{i, K(i)}\right\}$ is a partition of $\mathcal{I},{ }^{13} K(i)=\left|\mathcal{G}_{i}\right|$ is the number of groups in $\mathcal{G}_{i}$, and player $i$ is the only player in group 1, that is, $G_{i, 1}=\{i\}$.

2. Each player $i \in \mathcal{I}$ forms her beliefs as in definition 1 under the assumption that in each group in her group partition, every player in that group receives the same signal. That is, for every $j$ in group $G_{i, k}$,

$$
x_{i}^{j}=x+v \epsilon_{i, k}
$$

where the $\left\{\epsilon_{i, k}\right\}_{i \in \mathcal{I} ; k=1, \ldots, K(i)}$ are i.i.d., jointly independent with $f$, and drawn from $[-1,1]$ according to atomless, continuous, and symmetric density $\phi_{i, k}$. When convenient, we may write $x_{i}^{G_{k}}$ for $x_{i}^{j}$, for every $j$ in group $G_{i, k}$.

In other words, when $\mathcal{G}_{i}$ is the group partition for player $i$, player $i$ forms her beliefs under the assumption that each opponent in the same group of opponents $G_{i, k}$ receives the same information $x_{0}+v \epsilon_{i, k}$ about the true state. One extreme case is when each $\mathcal{G}_{i}$ is the discrete partition consisting of $I$ singletons, in which case player $i$ 's beliefs about the information received by others reduces to the earlier formulation of private, independent information used in theorem 1 . The other extreme case is when $\mathcal{G}_{i}$ consists of two elements only: $\{i\}$ and $\mathcal{I} \backslash\{i\}$. In this case, player $i$ stereotypes signals of all opponents into one group. As mentioned above, this may be a natural assumption in some cases, for example, when there are a large number of players and beliefs become more stereotypical under higher cognitive loads (Macrae and Bodenhausen (2000)).

From here on, to ease notational burden, and to provide a connection to theorem 1, we follow the convention that for player $i$ with group partition $\mathcal{G}_{i}$, we let $x_{i}=x_{i}^{i}$ denote the signal received by player $i$, and let $x_{-i}$ denote the vector of signals $\left(x_{i}^{j}\right)_{j \neq i}$ received by opponents of player $i$, and let $K=\max _{i \in \mathcal{I}} K(i)$ denote the maximum number of groups in the group partition for any player $i$.

\footnotetext{
${ }^{13} \mathrm{~A}$ collection of nonempty, disjoint sets whose union is $\mathcal{I}$.
} 
We can apply propositions 1 and 2 as before and establish the following relationships, analogous to equations (2) and (3). Suppose $K=2$. In this case, for all $\epsilon>0$, there exists $\bar{v}>0$ such that for all $v<\bar{v}$, and all $x_{i}, x_{j}(i \neq j)$ such that $B\left(x_{i}, 2 v\right) \subset X_{0}$ and $B\left(x_{j}, 2 v\right) \subset X_{0}$,

$$
F_{i}\left(x_{-i} \mid x_{i}, v\right)+F_{j}\left(x_{-j} \mid x_{j}, v\right) \geq 1-\epsilon .
$$

For $K \geq 2$, notice that for each player $i$, the conditional beliefs for all opponents are determined by the conditional joint distribution for the $K(i)-1$ groups of opponents in the group partition of player $i$. In particular, $F_{i}\left(x_{i} \mathbf{1}_{I-1} \mid x_{i}, v\right)=F_{i}^{\prime}\left(x_{i} \mathbf{1}_{K(i)-1} \mid x_{i}, v\right)$, where $F_{i}^{\prime}$ is the conditional distribution on the $(K(i)-1)$-dimensional $\left(x_{i}^{G_{k}}\right)_{k=2, \ldots, K(i)}$ derived from the joint distribution of $\left(x_{i}^{G_{k}}\right)_{k=1, \ldots, K(i)}$. Moreover, an argument similar to that after proposition 2 yields that for all $\epsilon>0$, there exists $\bar{v}>0$ such that for all $v<\bar{v}$, and all $x_{i}$ such that $B\left(x_{i}, 2 v\right) \subset X_{0}, F_{i}^{\prime}\left(x_{i} \mathbf{1}_{K(i)-1} \mid x_{i}, v\right) \geq \frac{1}{K(i)}-\epsilon$. Therefore, it follows that

$$
F_{i}\left(x_{i} \mathbf{1}_{I-1} \mid x_{i}, v\right) \geq \frac{1}{K(i)}-\epsilon .
$$

We now come to theorem 3 .

Theorem 3. Let $\Gamma$ be a monotone global game that has a dominance region for a profile of actions $a^{*}, \mathcal{G}$ be a group partition system, and let

$$
\hat{X}= \begin{cases}\left\{x \in X_{0} \mid \forall i \neq j, p_{i}^{a_{i}^{*}}(x)+p_{j}^{a_{j}^{*}}(x)<1\right\} & \text { if } K=2, \\ \left\{x \in X_{0} \mid \forall i \in \mathcal{I}, p_{i}^{a_{i}^{*}}(x)<\frac{1}{K(i)}\right\} & \text { if } K>2 .\end{cases}
$$

For every open interval $\mathcal{O} \subset \hat{X}$ such that either $\mathcal{O} \cap(-\infty, \underline{x}] \neq \emptyset$, or $\mathcal{O} \cap[\bar{x}, \infty) \neq$ $\emptyset$, (depending on which dominance region exists,) we have that $\forall x \in \mathcal{O}, \exists \hat{v}>0, \forall v \in$ $(0, \hat{v}], \forall s \in \mathcal{S}^{v}, s(x)=a^{*}$.

Proof. The case when $K>2$ follows immediately from theorem 1 after replacing the equation in claim 4 with equation 6 above.

Now consider the case when $K=2$. We can proceed along the exact lines of theorem 1 up to claim 3. In order to finish the proof, we must account for the fact that there are 
$I$ players, and thus the players who are associated with the smallest and second smallest points, $x_{\ell}^{v_{n}}$ and $x_{k}^{v_{n}}$, respectively, may differ for each $n$. To that end, notice that for each $\varepsilon>0, \exists N>0, \forall n \geq N$

$$
\max _{i \neq j}\left(p_{i}^{a_{i}^{*}}\left(x_{i}^{v_{n}}\right)+p_{j}^{a_{j}^{*}}\left(x_{j}^{v_{n}}\right)\right) \geq p_{\ell}^{a_{\ell}^{*}}\left(x_{\ell}^{v_{n}}\right)+p_{k}^{a_{k}^{*}}\left(x_{k}^{v_{n}}\right) \geq 1-\epsilon
$$

As in theorem 1 , we have that $x_{\ell}^{v_{n}}$ converges to some $x^{*}$ as $n \rightarrow \infty$, and hence $x_{k}^{v_{n}}$ does so as well. Also, since each $p_{i}^{a_{i}^{*}}$ is upper semi-continuous, it follows that each $p_{i}^{a_{i}^{*}}+p_{j}^{a_{j}^{*}}$ is jointly upper semi-continuous, and hence so is $\max _{i \neq j}\left(p_{i}^{a_{i}^{*}}+p_{j}^{a_{j}^{*}}\right)$. Thus, we have that

$$
\max _{i \neq j}\left(p_{i}^{a_{i}^{*}}\left(x^{*}\right)+p_{j}^{a_{j}^{*}}\left(x^{*}\right)\right) \geq 1
$$

If we allow $i, j \in \mathcal{I}$ to be those two players such that $p_{i}^{a_{i}^{*}}\left(x^{*}\right)+p_{j}^{a_{j}^{*}}\left(x^{*}\right)=\max _{i \neq j}\left(p_{i}^{a_{i}^{*}}\left(x^{*}\right)+\right.$ $\left.p_{j}^{a_{j}^{*}}\left(x^{*}\right)\right)$, then $p_{i}^{a_{i}^{*}}\left(x^{*}\right)+p_{j}^{a_{j}^{*}}\left(x^{*}\right) \geq 1$, contradicting the fact that $p_{i}^{a_{i}^{*}}\left(x^{*}\right)+p_{j}^{a_{j}^{*}}\left(x^{*}\right)<1$.

Notice that theorems 2 and 3 may be combined to formulate a result for subjective group partitions in which there are multiple players in the group of player $i$.

Consider the following examples.

Example 5 (Market Entry). Consider the following simplified version of Brander and Spencer (1985), where a foreign firm $\left(F_{f}\right)$ decides whether to remain in $(R)$ or leave $(L)$ a market consisting of two domestic firms $F_{d}^{1}$ and $F_{d}^{2}$ ), both of whom must decide whether to enter $(E)$ or stay out $(S)$ of the market. The domestic firms receive a government subsidy $s \geq 0$, while the foreign firm does not. Consider the following payoffs.

$$
\begin{aligned}
& \begin{array}{ccc}
R & F_{f} & L \\
F_{d}^{2} & & F_{d}^{2}
\end{array}
\end{aligned}
$$

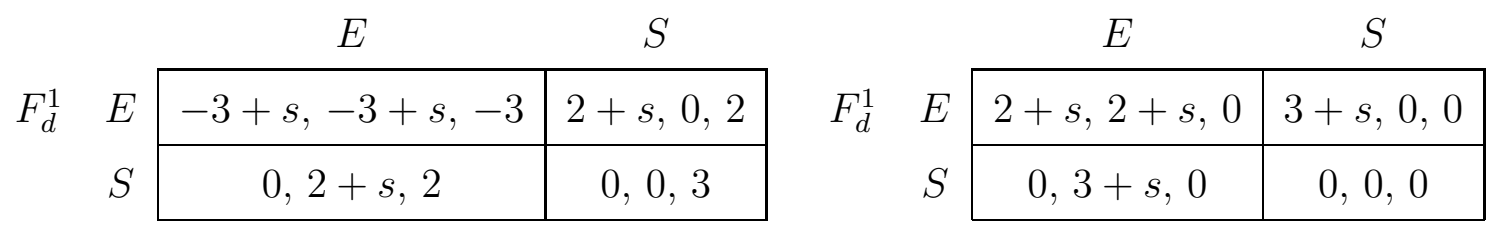

This is a game of strategic substitutes parameterized by $s \geq 0$. For $s \in[0,3]$, the Nash equilibria are given by $(E, S, R),(S, E, R)$, and $(E, E, L)$, and for $s>3,(E, E, L)$ is 
a strict Nash equilibrium, where $E$ is strictly dominant for the two domestic firms. Note that the parameter restriction $s \geq 0$ prevents us from establishing a lower dominance region, and symmetry in domestic firms implies no overlapping dominance regions. Thus FMP and Harrison and Jara-Moroni (2016) cannot be applied. In order to apply theorem 2 to the equilibrium $(E, E, L)$, we require that for each $i \in \mathcal{I}$,

$$
p_{i}^{a_{i}^{*}}<\frac{1}{3}
$$

Calculating the $p_{i}^{a_{i}^{*}}$ functions gives, $\forall s \geq 0$,

$$
\begin{gathered}
p_{1}^{a_{1}^{*}}(s)=p_{2}^{a_{2}^{*}}(s)=\left\{\begin{array}{cl}
\frac{3-s}{5} & 0 \leq s<3 \\
0 & s \geq 3
\end{array}\right. \\
p_{3}^{a_{3}^{*}}(s)=\frac{1}{2} .
\end{gathered}
$$

Notice that theorem 1 cannot be applied, because $p_{3}^{a_{3}^{*}}(s)>\frac{1}{3}$. However, if players have stereotypical beliefs, so that each player believes that opponents receive the same information, then each $\mathcal{G}_{i}$ consists of two elements and $G=2$. In this case,

$$
p_{i}^{a_{i}^{*}}(s)+p_{j}^{a_{j}^{*}}(s)<1
$$

holds for all $s>\frac{1}{2}$, and by theorem 2 , multiplicity is resolved on $\left(\frac{1}{2}, 3\right]$.

Example 6 (Strategic heterogeneity). Our framework allows for games in which some players exhibit strategic complements and others exhibit strategic substitutes at the same $x$. This is the case of strategic heterogeneity. Consider the investment choices in example 3 above. Consider a three player game, each with the same two actions $L$ and H. Payoffs may be interpreted similarly.

$$
\begin{aligned}
& \begin{array}{lll}
L & P 3
\end{array} \\
& \text { P2 P2 }
\end{aligned}
$$

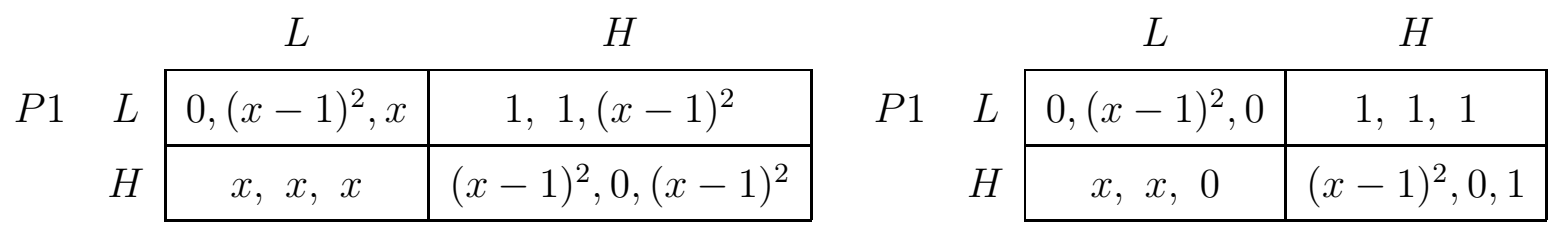


Notice that for $x<0, \mathrm{P} 1$ and $\mathrm{P} 2$ have strategic complements at $x, \mathrm{P} 3$ has strategic substitutes at $x$, and the game has two strict Nash equilibria, $(L, L, H)$ and $(H, H, L)$. For $x \in(0,2), \mathrm{P} 1$ and $\mathrm{P} 2$ have strategic substitutes at $x, \mathrm{P} 3$ has strategic complements at $x$, and there are two strict Nash equilibria, $(H, L, L)$ and $(L, H, H)$. For $x>2$, there is a strictly dominant equilibrium $(H, L, L)$. Notice that there is only one dominance region. Moreover, for every $x<2$, no game is GSC or GSC, all games are GSH.

Our results apply to this situation. The example is made tractable by exploiting symmetry in payoffs to yield $p_{1}^{H}(x), p_{2}^{L}(x)$, and $p_{3}^{L}(x)$ as follows.

$$
p_{1}^{H}(x)=p_{2}^{L}(x)=p_{3}^{L}(x)\left\{\begin{array}{cl}
\frac{1-(x-1)^{2}}{1-(x-1)^{2}+x} & 0<x<2 \\
0 & x \geq 2
\end{array}\right.
$$

For $x \in(0,2), p_{1}(x)<\frac{1}{3}$ is satisfied for all $x>\frac{3}{2}$. Thus, multiplicity is resolved on $\left(\frac{3}{2}, 2\right]$. With group partitions, this range may be extended back to $(1,2]$. 


\section{References}

Baliga, S., And T. Sjostrom (2012): "The Strategy of Manipulating Conflict," American Economic Review, 102, 2897-2922.

Basteck, C., T. R. Daniëls, and F. Heinemann (2013): "Characterising equilibrium selection in global games with strategic complementarities," Journal of Economic Theory, 148(6), 2620-2637.

Brander, J. A., And B. J. Spencer (1985): "Export subsidies and international market share rivalry," Journal of international Economics, 18(1-2), 83-100.

Carlsson, H., and E. van Damme (1993): "Global Games and Equilibrium Selection," Econometrica, 61(5), 989-1018.

Frankel, D. M., S. Morris, And A. Pauzner (2003): "Equilibrium selection in global games with strategic complementarities," Journal of Economic Theory, 108(1), 1-44.

Harrison, R., AND P. JARA-Moroni (2016): "Equilibrium selection in global games with strategic substitutes," Working paper.

HeAly, P. J. (2007): "Group reputations, stereotypes, and cooperation in a repeated labor market," American Economic Review, 97(5), 1751-1773.

KaJII, A., AND S. Morris (1997): "The Robustness of Equilibria to Incomplete Information," Econometrica, 65(6), 1283-1309.

Keser, C., I. Suleymanova, and C. Wey (2012): "Technology adoption in markets with network effects: Theory and experimental evidence," Information Economics and Policy, 24(3-4), 262-276.

Kilgour, D. M., And F. C. Zagare (1991): "Credibility, uncertainty, and deterrence," American Journal of Political Science, 35(2), 305-334.

Macrae, C. N., and G. V. Bodenhausen (2000): "Social cognition: Thinking categorically about others," Annual Review of Psychology, 51(1), 93-120.

Morris, S., R. Rob, And H. S. Shin (1995): "p-Dominance and Belief Potential," Econometrica, 63(1), 145-157.

Morris, S., AND H. S. SHIN (2005): "Notes on strategic substitutes and complements in global games," Available online at author's website.

Weinstein, J., AND M. YILDIZ (2007): "A structure theorem for rationalizability with application to robust predictions of refinements," Econometrica, 75(2), 365-400. 


\section{Appendix}

We now prove proposition 1.

Proof. (of Proposition 1)

The first assertion is given in CvD (1993) and is thus omitted.

For the second assertion, recall for each player $i$, and each signal $x_{i}, f_{i}^{*}\left(\cdot \mid x_{i}, v\right)$ is defined as in equation 1 . In particular, because $x_{i} \in \mathcal{I}$, the denominator equals 1 , and thus

$$
f_{i}^{*}\left(x_{-i} \mid x_{i}, v\right)=\int_{\mathbb{R}} \tilde{\phi}_{i}^{v, *}\left(x_{i}-x\right) \prod_{j \neq i} \tilde{\phi}_{j}^{v, *}\left(x_{j}-x\right) d x
$$

Since each $\tilde{\phi}_{j}^{v, *}\left(x_{j}-x\right)=\frac{1}{2 v}$ on $\left[x_{j}-v, x_{j}+v\right]$ and zero elsewhere, we then have that

$$
f_{i}^{*}\left(x_{-i} \mid x_{i}, v\right)=\left(\frac{1}{2 v}\right)^{I} m\left(\bigcap_{j \in \mathcal{I}}\left[x_{j}-v, x_{j}+v\right]\right) .
$$

To make the calculation $F_{i}^{*}\left(x_{i} \mathbf{1}_{I-1} \mid x_{i}, v\right)$, consider vectors of the form $x_{i} \mathbf{1}_{I-1}-z$ for $z \in[0,2 v)^{I-1}$. By the equation above we have that whenever $a_{m}=\max _{j \neq i}\left(a_{j}\right)$,

$$
f_{i}^{*}\left(x_{i} \mathbf{1}_{I-1}-z \mid x_{i}, v\right)=\left(\frac{1}{2 v}\right)^{I}\left(2 v-a_{m}\right) .
$$

Consider any $k \in \mathcal{I} \backslash\{i\}$, and arbitrarily enumerate the rest of the $j \neq i, k$. Then the probability that player $i$ receives the highest signal, followed by player 1 , and so on all the way to player $k$ can be written as

$\operatorname{Pr}\left(\left\{x_{i}-2 v<x_{k}<\cdots<x_{2}<x_{1}<x_{i}\right\} \mid x_{i}, v\right)=\int_{\left\{x_{i}-2 v<x_{k}<\cdots<x_{2}<x_{1}<x_{i}\right\}} f_{i}^{*}\left(x_{-i} \mid x_{i}, v\right) d x_{-i}$,

which, by a change of variables and the above observation, can be written as

$$
\int_{\left\{0<a_{1}<a_{2} \cdots<a_{k}<2 v\right\}} f_{i}^{*}\left(x_{i} \mathbf{1}_{I-1}-a \mid x_{i}, v\right) d a_{-i}=\int_{0}^{2 v} \int_{0}^{a_{k}} \cdots \int_{0}^{a_{2}}\left(\frac{1}{2 v}\right)^{N}\left(2 v-a_{k}\right) \prod_{j=1}^{k} d a_{j} .
$$

For any such $k$, there are $(I-2)$ ! enumerations of the other $j \neq i, k$ players, and thus the probability that player $i$ receives the largest signal followed by player $k$ is given by

$$
(I-2) ! \int_{0}^{2 v} \int_{0}^{a_{k}} \cdots \int_{0}^{a_{2}}\left(\frac{1}{2 v}\right)^{I}\left(2 v-a_{k}\right) \prod_{j=1}^{k} d a_{j} .
$$


Finally, because there are $(I-1)$ opponents who could receive the second highest signal after player $i$, we have that

$$
\begin{gathered}
F_{i}^{*}\left(x_{i} \mathbf{1}_{I-1} \mid x_{i}, v\right) \geq(I-1)(I-2) ! \int_{0}^{2 v} \int_{0}^{a_{k}} \cdots \int_{0}^{a_{2}}\left(\frac{1}{2 v}\right)^{N}\left(2 v-a_{j}\right) \prod_{j=1}^{k} d a_{j} \\
=(I-1) ! \int_{0}^{2 v} \int_{0}^{a_{k}} \cdots \int_{0}^{a_{2}}\left(\frac{1}{2 v}\right)^{I}\left(2 v-a_{k}\right) \prod_{j=1}^{k} d a_{j}=\frac{1}{I}
\end{gathered}
$$

We now proceed to prove proposition 6 . In order to do so, we first prove propositions 812 below. Proposition 13 concludes the proof. Recall from the discussion on $p$-dominance that for a profile of actions $a^{*}$, for each player $i$, and for each $x \in \mathbb{R}$,

$$
p_{i}^{a_{i}^{*}}(x)=\inf \left\{\xi \in[0,1] \mid \forall \lambda_{-i} \in \mathcal{M}_{-i}, \lambda_{-i}\left(a_{-i}^{*}\right) \geq \xi \Rightarrow \forall a_{i}^{\prime} \neq a_{i}^{*}, \Delta \mathcal{U}_{i}\left(a_{i}^{*}, a_{i}^{\prime}, \lambda_{-i}, x\right) \geq 0\right\} .
$$

For notational convenience, let $E$ denote the set over which infimum is taken; that is, $p_{i}^{a_{i}^{*}}(x)=\inf E$. Recall that if $a_{i}^{*}$ is a best response of player $i$ to $a_{-i}^{*}$ at $x$, then $1 \in E$ and therefore, $p_{i}^{a_{i}^{*}}(x)$ is well-defined. Moreover, $E$ satisfies the property that $\xi \in E \Rightarrow[\xi, 1] \subset$ $E$. It will be convenient to deconstruct this computation some more. For each player $i$ and for each $a_{i}^{\prime} \neq a_{i}^{*}$, let

$$
p_{i}^{a_{i}^{*}}\left(x, a_{i}^{\prime}\right)=\inf \left\{\xi \in[0,1] \mid \forall \lambda_{-i} \in \mathcal{M}_{-i}, \lambda_{-i}\left(a_{-i}^{*}\right) \geq \xi \Rightarrow \Delta \mathcal{U}_{i}\left(a_{i}^{*}, a_{i}^{\prime}, \lambda_{-i}, x\right) \geq 0\right\} .
$$

For notational convenience, let $E\left(a_{i}^{\prime}\right)$ denote the set over which infimum is taken. In other words, $p_{i}^{a_{i}^{*}}\left(x, a_{i}^{\prime}\right)=\inf E\left(a_{i}^{\prime}\right)$. As earlier, if $a_{i}^{*}$ is a best response of player $i$ to $a_{-i}^{*}$ at $x$, then $1 \in E\left(a_{i}^{\prime}\right)$ and therefore, $p_{i}^{a_{i}^{*}}\left(x, a_{i}^{\prime}\right)$ is well-defined. Moreover, $E\left(a_{i}^{\prime}\right)$ satisfies the property that $\xi \in E\left(a_{i}^{\prime}\right) \Rightarrow[\xi, 1] \subset E\left(a_{i}^{\prime}\right)$. Here are some useful connections between these two formulations.

Proposition 8. Let $\Gamma$ be a global game and $a^{*}$ a profile of actions.

For $i \in \mathcal{I}$, if $a_{i}^{*}$ is the unique best response of player $i$ to $a_{-i}^{*}$ at $x$, then

1. For every $a_{i}^{\prime} \neq a_{i}^{*}, E\left(a_{i}^{\prime}\right)=\left[p_{i}^{a_{i}^{*}}\left(x, a_{i}^{\prime}\right), 1\right]$

2. $E=\left[p_{i}^{a_{i}^{*}}(x), 1\right]$

3. $p_{i}^{a_{i}^{*}}(x)=\max _{a_{i}^{\prime} \neq a_{i}^{*}} p_{i}^{a_{i}^{*}}\left(x, a_{i}^{\prime}\right)$

Proof. To prove statement (1), it is sufficient to show that $p_{i}^{a_{i}^{*}}\left(x, a_{i}^{\prime}\right) \in E\left(a_{i}^{\prime}\right)$. If $p_{i}^{a_{i}^{*}}\left(x, a_{i}^{\prime}\right)=1$, then we are done, because $1 \in E\left(a_{i}^{\prime}\right)$. Suppose $p_{i}^{a_{i}^{*}}\left(x, a_{i}^{\prime}\right)<1$. Fix $\lambda_{-i} \in \mathcal{M}_{-i}$ arbitrarily and suppose $\lambda_{-i}\left(a_{-i}^{*}\right) \geq p_{i}^{a^{*}}\left(x, a_{i}^{\prime}\right)$. As case 1 , suppose $\lambda_{-i}\left(a_{-i}^{*}\right)>$ 
$p_{i}^{a_{i}^{*}}\left(x, a_{i}^{\prime}\right)$. Then there is $\xi \in E\left(a_{i}^{\prime}\right)$ such that $\lambda_{-i}\left(a_{-i}^{*}\right)>\xi>p_{i}^{a_{i}^{*}}\left(x, a_{i}^{\prime}\right)$, and consequently, $\Delta \mathcal{U}_{i}\left(a_{i}^{*}, a_{i}^{\prime}, \lambda_{-i}, x\right) \geq 0$. As case 2 , suppose $\lambda_{-i}\left(a_{-i}^{*}\right)=p_{i}^{a_{i}^{*}}\left(x, a_{i}^{\prime}\right)<1$. Then support of $\lambda_{-i}$ has an element other than $a_{-i}^{*}$. Moving some of the probability of this element to $a_{-i}^{*}$, we may form a sequence $\lambda_{-i}^{n} \in \mathcal{M}_{-i}$ such that for all $n \geq 1, \lambda_{-i}^{n}\left(a_{-i}^{*}\right)>\lambda_{-i}\left(a_{-i}^{*}\right)=p_{i}^{a_{i}^{*}}\left(x, a_{i}^{\prime}\right)$ and $\lambda_{-i}^{n} \rightarrow \lambda_{-i}$. Now, by case 1 , for all $n \geq 1, \Delta \mathcal{U}_{i}\left(a_{i}^{*}, a_{i}^{\prime}, \lambda_{-i}^{n}, x\right) \geq 0$ and by continuity of expected utility in opponents' strategies, it follows that $\Delta \mathcal{U}_{i}\left(a_{i}^{*}, a_{i}^{\prime}, \lambda_{-i}, x\right) \geq 0$. In both cases, we conclude that $\Delta \mathcal{U}_{i}\left(a_{i}^{*}, a_{i}^{\prime}, \lambda_{-i}, x\right) \geq 0$, whence $p_{i}^{a_{i}^{*}}\left(x, a_{i}^{\prime}\right) \in E\left(a_{i}^{\prime}\right)$. The proof of statement (2) is similar.

To prove statement (3), fix $\lambda_{-i} \in \mathcal{M}_{-i}$ arbitrarily and suppose $\lambda_{-i}\left(a_{-i}^{*}\right) \geq \max _{a_{i}^{\prime} \neq a_{i}^{*}} p_{i}^{a_{i}^{*}}\left(x, a_{i}^{\prime}\right)$. Then $\forall a_{i}^{\prime} \neq a_{i}^{*}, \lambda_{-i}\left(a_{-i}^{*}\right) \geq p_{i}^{a_{i}^{*}}\left(x, a_{i}^{\prime}\right)$, which implies that $\forall a_{i}^{\prime} \neq a_{i}^{*}, \Delta \mathcal{U}_{i}\left(a_{i}^{*}, a_{i}^{\prime}, \lambda_{-i}, x\right) \geq 0$. As $\lambda_{-i}$ is arbitrary, it follows that $\max _{a_{i}^{\prime} \neq a_{i}^{*}} p_{i}^{a_{i}^{*}}\left(x, a_{i}^{\prime}\right) \in E$, whence $p_{i}^{a_{i}^{*}}(x) \leq \max _{a_{i}^{\prime} \neq a_{i}^{*}} p_{i}^{a_{i}^{*}}\left(x, a_{i}^{\prime}\right)$. In the other direction, notice that for an arbitrary $\lambda_{-i} \in \mathcal{M}_{-i}, \lambda_{-i}\left(a_{-i}^{*}\right) \geq p_{i}^{a_{i}^{*}}(x) \Rightarrow \forall a_{i}^{\prime} \neq$ $a_{i}^{*}, \Delta \mathcal{U}_{i}\left(a_{i}^{*}, a_{i}^{\prime}, \lambda_{-i}, x\right) \geq 0$, which implies that $\forall a_{i}^{\prime} \neq a_{i}^{*}, p_{i}^{a_{i}^{*}}(x) \in E\left(a_{i}^{\prime}\right)$. Consequently, $\forall a_{i}^{\prime} \neq a_{i}^{*}, p_{i}^{a_{i}^{*}}(x) \geq p_{i}^{a_{i}^{*}}\left(x, a_{i}^{\prime}\right)$, whence $p_{i}^{a_{i}^{*}}(x) \geq \max _{a_{i}^{\prime} \neq a_{i}^{*}} p_{i}^{a_{i}^{*}}\left(x, a_{i}^{\prime}\right)$.

Proposition 9. Let $\Gamma$ be a global game and $a^{*}$ a profile of actions. For $i \in \mathcal{I}$, if $a_{i}^{*}$ is the unique best response of player $i$ to $a_{-i}^{*}$ at $x$, then

$$
\forall a_{i}^{\prime} \neq a_{i}^{*}, \forall \lambda_{-i} \in \mathcal{M}_{-i}, \lambda_{-i}\left(a_{-i}^{*}\right)>p_{i}^{a_{i}^{*}}\left(x, a_{i}^{\prime}\right) \Rightarrow \Delta \mathcal{U}_{i}\left(a_{i}^{*}, a_{i}^{\prime}, \lambda_{-i}, x\right)>0 .
$$

Proof. Suppose the hypothesis and fix $\hat{\lambda}_{-i} \in \mathcal{M}_{-i}$ arbitrarily such that $\hat{\lambda}_{-i}\left(a_{-i}^{*}\right)>$ $p_{i}^{a_{i}^{*}}\left(x, a_{i}^{\prime}\right)$. Notice that if $\hat{\lambda}_{-i}\left(a_{-i}^{*}\right)=1$, then $\Delta \mathcal{U}_{i}\left(a_{i}^{*}, a_{i}^{\prime}, \hat{\lambda}_{-i}, x\right)=\Delta u_{i}\left(a_{i}^{*}, a_{i}^{\prime}, a_{-i}^{*}, x\right)>0$ by the fact that $a_{i}^{*}$ is the unique best response of player $i$ to $a_{-i}^{*}$ at $x$, and we are done. So suppose $\hat{\lambda}_{-i}\left(a_{-i}^{*}\right)<1$.

Let $B=\arg \min _{a_{-i}} \Delta u_{i}\left(a_{i}^{*}, a_{i}^{\prime}, a_{-i}, x\right)$. As each player has finitely many actions, $B$ is non-empty and finite. Let $|B|$ denote the number of elements in $B$, and denote by $a_{-i}^{\min }$ an arbitrarily fixed point in $B$.

As case 1 , suppose $a_{-i}^{*} \in B$. Then $\Delta \mathcal{U}_{i}\left(a_{i}^{*}, a_{i}^{\prime}, \hat{\lambda}_{-i}, x\right) \geq \Delta u_{i}\left(a_{i}^{*}, a_{i}^{\prime}, a_{-i}^{*}, x\right)>0$, where the strict inequality is implied by the assumption that $a_{i}^{*}$ is the unique best response of player $i$ to $a_{-i}^{*}$ at $x$. In this case, we are done. As case 2 , suppose $a_{-i}^{*} \notin B$. Define $\lambda_{-i}^{\prime}$ as follows: $\lambda_{-i}^{\prime}\left(a_{-i}\right)=\hat{\lambda}_{-i}\left(a_{-i}^{*}\right)$, if $a_{-i}=a_{-i}^{*}$, and $\lambda_{-i}^{\prime}\left(a_{-i}\right)=\frac{1-\hat{\lambda}_{-i}\left(a_{-i}^{*}\right)}{|B|}$, if $a_{-i} \in B$, and $\lambda_{-i}^{\prime}\left(a_{-i}\right)=0$ otherwise. Then

$$
\begin{aligned}
\Delta \mathcal{U}_{i}\left(a_{i}^{*}, a_{i}^{\prime}, \hat{\lambda}_{-i}, x\right) & =\Delta u_{i}\left(a_{i}^{*}, a_{i}^{\prime}, a_{-i}^{*}, x\right) \hat{\lambda}_{-i}\left(a_{-i}^{*}\right)+\sum_{a_{-i} \neq a_{-i}^{*}} \Delta u_{i}\left(a_{i}^{*}, a_{i}^{\prime}, a_{-i}, x\right) \hat{\lambda}_{-i}\left(a_{-i}\right) \\
& \geq \Delta u_{i}\left(a_{i}^{*}, a_{i}^{\prime}, a_{-i}^{*}, x\right) \hat{\lambda}_{-i}\left(a_{-i}^{*}\right)+\sum_{a_{-i} \neq a_{-i}^{*}} \Delta u_{i}\left(a_{i}^{*}, a_{i}^{\prime}, a_{-i}^{\min }, x\right) \hat{\lambda}_{-i}\left(a_{-i}\right) \\
& =\Delta u_{i}\left(a_{i}^{*}, a_{i}^{\prime}, a_{-i}^{*}, x\right) \lambda_{-i}^{\prime}\left(a_{-i}^{*}\right)+\sum_{a_{-i} \in B} \Delta u_{i}\left(a_{i}^{*}, a_{i}^{\prime}, a_{-i}, x\right) \lambda_{-i}^{\prime}\left(a_{-i}\right) \\
& =\Delta \mathcal{U}_{i}\left(a_{i}^{*}, a_{i}^{\prime}, \lambda_{-i}^{\prime}, x\right) .
\end{aligned}
$$

Now let $\epsilon>0$ be such that $p_{i}^{a_{i}^{*}}\left(x, a_{i}^{\prime}\right)+\epsilon<\hat{\lambda}_{-i}\left(a_{-i}^{*}\right)$. Define $\lambda_{-i}^{\prime \prime}$ as follows: $\lambda_{-i}^{\prime \prime}\left(a_{-i}\right)=$ 
$p_{i}^{a_{i}^{*}}\left(x, a_{i}^{\prime}\right)+\epsilon$, if $a_{-i}=a_{-i}^{*}$, and $\lambda_{-i}^{\prime \prime}\left(a_{-i}\right)=\frac{1-p_{i}^{a_{i}^{*}}\left(x, a_{i}^{\prime}\right)-\epsilon}{|B|}$, if $a_{-i} \in B$, and $\lambda_{-i}^{\prime \prime}\left(a_{-i}\right)=0$ otherwise. Then $\lambda_{-i}^{\prime \prime}\left(a_{-i}^{*}\right)>p_{i}^{a_{i}^{*}}\left(x, a_{i}^{\prime}\right)$ and by a previous result, it follows that $\Delta \mathcal{U}_{i}\left(a_{i}^{*}, a_{i}^{\prime}, \lambda_{-i}^{\prime \prime}, x\right) \geq$ 0. Moreover,

$$
\begin{aligned}
\Delta \mathcal{U}_{i}\left(a_{i}^{*}, a_{i}^{\prime}, \lambda_{-i}^{\prime}, x\right) & =\Delta u_{i}\left(a_{i}^{*}, a_{i}^{\prime}, a_{-i}^{*}, x\right) \hat{\lambda}_{-i}\left(a_{-i}^{*}\right)+\sum_{a_{-i} \in B} \Delta u_{i}\left(a_{i}^{*}, a_{i}^{\prime}, a_{-i}, x\right) \frac{1-\hat{\lambda}_{-i}\left(a_{-i}^{*}\right)}{|B|} \\
& =\left[\Delta u_{i}\left(a_{i}^{*}, a_{i}^{\prime}, a_{-i}^{*}, x\right)-\Delta u_{i}\left(a_{i}^{*}, a_{i}^{\prime}, a_{-i}^{\min }, x\right)\right] \hat{\lambda}\left(a_{-i}^{*}\right)+\Delta u_{i}\left(a_{i}^{*}, a_{i}^{\prime}, a_{-i}^{\min }, x\right) \\
& >\left[\Delta u_{i}\left(a_{i}^{*}, a_{i}^{\prime}, a_{-i}^{*}, x\right)-\Delta u_{i}\left(a_{i}^{*}, a_{i}^{\prime}, a_{-i}^{\min }, x\right)\right]\left(p_{i}^{a_{i}^{*}}\left(x, a_{i}^{\prime}\right)+\epsilon\right)+\Delta u_{i}\left(a_{i}^{*}, a_{i}^{\prime}, a_{-i}^{\min }, x\right) \\
& =\Delta u_{i}\left(a_{i}^{*}, a_{i}^{\prime}, a_{-i}^{*}, x\right)\left(p_{i}^{a_{i}^{*}}\left(x, a_{i}^{\prime}\right)+\epsilon\right)+\sum_{a_{-i} \in B} \Delta u_{i}\left(a_{i}^{*}, a_{i}^{\prime}, a_{-i}, x\right) \frac{1-p_{i}^{a_{i}}\left(x, a_{i}^{\prime}\right)-\epsilon}{|B|} \\
& =\Delta \mathcal{U}_{i}\left(a_{i}^{*}, a_{i}^{\prime}, \lambda_{-i}^{\prime \prime}, x\right) \geq 0
\end{aligned}
$$

where the strict inequality follows because $a_{-i}^{\min } \in B$ and $a_{-i}^{*} \notin B$. Therefore, $\Delta \mathcal{U}_{i}\left(a_{i}^{*}, a_{i}^{\prime}, \hat{\lambda}_{-i}, x\right)>$ 0 , as desired.

Similarly, for player $i$ and for $a_{i}^{\prime} \neq a_{i}^{*}$, let $D_{i}^{a_{i}^{*}}\left(a_{i}^{\prime}\right)$ be the set of states where $a_{i}^{*}$ strictly dominates $a_{i}^{\prime}$ for player $i$. That is,

$$
D_{i}^{a_{i}^{*}}\left(a_{i}^{\prime}\right)=\left\{x \in \mathbb{R} \mid \forall a_{-i} \in A_{-i}, \Delta u_{i}\left(a_{i}^{*}, a_{i}^{\prime}, a_{-i}, x\right)>0\right\} .
$$

As $u_{i}$ is continuous in $x$ and each player has finitely many actions, it follows that $D_{i}^{a_{i}^{*}}\left(a_{i}^{\prime}\right)$ is an open set. Also notice that for each $x, x \in D_{i}^{a_{i}^{*}}\left(a_{i}^{\prime}\right) \Rightarrow p_{i}^{a_{i}^{*}}\left(x, a_{i}^{\prime}\right)=0$.

For player $i$ and for $a_{i}^{\prime} \neq a_{i}^{*}$, consider the correspondence $\psi: \mathbb{R} \rightrightarrows \mathcal{M}_{-i}$ given by

$$
\psi(x)=\left\{\lambda_{-i} \in \mathcal{M}_{-i} \mid \Delta \mathcal{U}_{i}\left(a_{i}^{*}, a_{i}^{\prime}, \lambda_{-i}, x\right)=0\right\},
$$

and the continuous function $f: \mathcal{M}_{-i} \rightarrow \mathbb{R}$ given by $f\left(\lambda_{-i}\right)=\lambda_{-i}\left(a_{-i}^{*}\right)$. We have the following result.

Proposition 10. Let $\Gamma$ be a global game and $a^{*}$ a profile of actions. For every $x \in \mathbb{R}$, for every player $i$, and every $a_{i}^{\prime} \neq a_{i}^{*}$,

1. $\psi(x) \neq \emptyset \Rightarrow x \notin D_{i}^{a_{i}^{*}}\left(a_{i}^{\prime}\right)$.

2. If $a_{i}^{*}$ is a best response of player $i$ to $a_{-i}^{*}$ at $x$, then $x \notin D_{i}^{a_{i}^{*}}\left(a_{i}^{\prime}\right) \Rightarrow \psi(x) \neq \emptyset$.

3. $\psi(x) \neq \emptyset \Rightarrow \sup _{\lambda_{-i} \in \psi(x)} f\left(\lambda_{-i}\right)$ is well-defined and achieved on $\psi(x)$.

Proof. To prove statement (1), consider the contrapositive and suppose $x \in D_{i}^{a_{i}^{*}}\left(a_{i}^{\prime}\right)$. Then $\forall \lambda_{-i} \in \mathcal{M}_{-i}, \Delta \mathcal{U}_{i}\left(a_{i}^{*}, a_{i}^{\prime}, \lambda_{-i}, x\right)>0$, whence $\psi(x)=\emptyset$.

To prove statement (2), suppose $a_{i}^{*}$ is a best response of player $i$ to $a_{-i}^{*}$ at $x$. Suppose $x \notin D_{i}^{a_{i}^{*}}\left(a_{i}^{\prime}\right)$. Then there is a profile of opponent actions $\hat{a}_{-i}$ such that $\Delta u_{i}\left(a_{i}^{*}, a_{i}^{\prime}, \hat{a}_{-i}, x\right) \leq$ 
0. Consider the following parameterized mixed strategies in $\mathcal{M}_{-i}$; for $\alpha \in[0,1], \lambda_{-i}^{\alpha}=$ $\alpha \delta_{a_{-i}^{*}}+(1-\alpha) \delta_{\hat{a}_{-i}}$, where $\delta_{a_{-i}^{*}}$ is the unit atom at $a_{-i}^{*}$ and $\delta_{\hat{a}_{-i}}$ is the unit atom at $\hat{a}_{-i}$. In this case, $\Delta \mathcal{U}_{i}\left(a_{i}^{*}, a_{i}^{\prime}, \lambda_{-i}^{0}, x\right) \leq 0$, and moreover, $a_{i}^{*}$ is a best response of player $i$ to $a_{-i}^{*}$ at $x$ implies that $\Delta \mathcal{U}_{i}\left(a_{i}^{*}, a_{i}^{\prime}, \lambda_{-i}^{1}, x\right) \geq 0$. By continuity of expected utility in mixed strategy profiles of opponents and the intermediate value theorem, there is $\hat{\alpha} \in(0,1)$ such that $\Delta \mathcal{U}_{i}\left(a_{i}^{*}, a_{i}^{\prime}, \lambda_{-i}^{\hat{\alpha}}, x\right)=0$. Consequently, $\lambda_{-i}^{\hat{\alpha}} \in \psi(x)$.

To prove statement (3), suppose $\psi(x)$ is not empty. Continuity of $\Delta \mathcal{U}_{i}$ in $\lambda_{-i}$ implies that $\psi(x)$ is a closed subset of a compact set, hence nonempty and compact. Moreover, $f$ is a continuous function, and therefore, $\sup _{\lambda_{-i} \in \psi(x)} f\left(\lambda_{-i}\right)$ is well-defined and achieved on $\psi(x)$.

For player $i$, for each $x \in \mathbb{R}$, and for each $a_{i}^{\prime} \neq a_{i}^{*}$, if $\psi(x)=\emptyset$, define $q_{i}^{a_{i}^{*}}\left(x, a_{i}^{\prime}\right)=0$, and if $\psi(x) \neq \emptyset$, define

$$
\begin{aligned}
q_{i}^{a_{i}^{*}}\left(x, a_{i}^{\prime}\right) & =\sup _{\lambda_{-i} \in \psi(x)} f\left(\lambda_{-i}\right) \\
& =\sup \left\{\lambda_{-i}\left(a_{-i}^{*}\right) \mid \lambda_{-i} \in \mathcal{M}_{-i} \text { and } \Delta \mathcal{U}_{i}\left(a_{i}^{*}, a_{i}^{\prime}, \lambda_{-i}, x\right)=0\right\}
\end{aligned}
$$

Proposition 10 shows that $\psi(x) \neq \emptyset \Rightarrow q_{i}^{a_{i}^{*}}\left(x, a_{i}^{\prime}\right)$ is well-defined and is achieved on $\psi(x)$.

Proposition 11. Let $\Gamma$ be a global game and $a^{*}$ a profile of actions.

For each player $i$, if $a_{i}^{*}$ is the unique best response of player $i$ to $a_{-i}^{*}$ at $x$, then for every $a_{i}^{\prime} \neq a_{i}^{*}, q_{i}^{a_{i}^{*}}\left(x, a_{i}^{\prime}\right)=p_{i}^{a_{i}^{*}}\left(x, a_{i}^{\prime}\right)$.

Proof. Suppose $x \in D_{i}^{a_{i}^{*}}\left(a_{i}^{\prime}\right)$. Then $p_{i}^{a_{i}^{*}}\left(x, a_{i}^{\prime}\right)=0=q_{i}^{a_{i}^{*}}\left(x, a_{i}^{\prime}\right)$, as desired.

Now suppose $x \notin D_{i}^{a_{i}^{*}}\left(a_{i}^{\prime}\right)$. Then $a_{i}^{*}$ is a best response of player $i$ to $a_{-i}^{*}$ at $x$ implies that $\psi(x) \neq \emptyset$. We first show that $p_{i}^{a_{i}^{*}}\left(x, a_{i}^{\prime}\right) \leq q_{i}^{a_{i}^{*}}\left(x, a_{i}^{\prime}\right)$. It is sufficient to show that $q_{i}^{a_{i}^{*}}\left(x, a_{i}^{\prime}\right) \in$ $E\left(a_{i}^{\prime}\right)$. Fix $\hat{\lambda}_{-i} \in \mathcal{M}_{-i}$ and suppose $\hat{\lambda}_{-i}\left(a_{-i}^{*}\right) \geq q_{i}^{a_{i}^{*}}\left(x, a_{i}^{\prime}\right)$. Suppose $\Delta \mathcal{U}_{i}\left(a_{i}^{*}, a_{i}^{\prime}, \hat{\lambda}_{-i}, x\right)<0$. Consider the following parameterized mixed strategy profiles in $\mathcal{M}_{-i}$; for $\alpha \in[0,1], \lambda_{-i}^{\alpha}=$ $\alpha \delta_{a_{-i}^{*}}+(1-\alpha) \hat{\lambda}_{-i}$, where $\delta_{a_{-i}^{*}}$ is the unit atom at $a_{-i}^{*}$. Then $a_{i}^{*}$ is a best response of player $i$ to $a_{-i}^{*}$ at $x$ implies that $\Delta \mathcal{U}_{i}\left(a_{i}^{*}, a_{i}^{\prime}, \lambda_{-i}^{1}, x\right) \geq 0$, and by assumption, $\Delta \mathcal{U}_{i}\left(a_{i}^{*}, a_{i}^{\prime}, \lambda_{-i}^{0}, x\right)<$ 0 . By continuity of expected utility in mixed strategy profiles of opponents and the intermediate value theorem, there is $\hat{\alpha} \in(0,1]$ such that $\Delta \mathcal{U}_{i}\left(a_{i}^{*}, a_{i}^{\prime}, \lambda_{-i}^{\hat{\alpha}}, x\right)=0$. That is, $\lambda_{-i}^{\hat{\alpha}}\left(a_{-i}^{*}\right)$ is in the set over which the supremum is taken in the definition of $q_{i}^{a_{i}^{*}}\left(x, a_{i}^{\prime}\right)$. Consequently, $q_{i}^{a_{i}^{*}}\left(x, a_{i}^{\prime}\right) \geq \lambda_{-i}^{\hat{\alpha}}\left(a_{-i}^{*}\right)$. Indeed,

$$
\begin{aligned}
q_{i}^{a_{i}^{*}}\left(x, a_{i}^{\prime}\right) & \geq \lambda_{-i}^{\hat{\alpha}}\left(a_{-i}^{*}\right) \\
& =\hat{\alpha}+(1-\hat{\alpha}) \hat{\lambda}_{-i}\left(a_{-i}^{*}\right) \\
& >\hat{\alpha} \hat{\lambda}_{-i}\left(a_{-i}^{*}\right)+(1-\hat{\alpha}) \hat{\lambda}_{-i}\left(a_{-i}^{*}\right) \\
& =\hat{\lambda}_{-i}\left(a_{-i}^{*}\right),
\end{aligned}
$$


where the strict inequality follows because $\hat{\alpha} \in(0,1]$, and $\Delta \mathcal{U}_{i}\left(a_{i}^{*}, a_{i}^{\prime}, \hat{\lambda}_{-i}, x\right)<0$ implies that $\hat{\lambda}_{-i}\left(a_{-i}^{*}\right)<1$. This contradicts $\hat{\lambda}_{-i}\left(a_{-i}^{*}\right) \geq q_{i}^{a_{i}^{*}}\left(x, a_{i}^{\prime}\right)$. Consequently, $\Delta \mathcal{U}_{i}\left(a_{i}^{*}, a_{i}^{\prime}, \hat{\lambda}_{-i}, x\right) \geq$ 0 , and therefore, $q_{i}^{a_{i}^{*}}\left(x, a_{i}^{\prime}\right) \in E\left(a_{i}^{\prime}\right)$, as desired.

We now show that $p_{i}^{a_{i}^{*}}\left(x, a_{i}^{\prime}\right) \geq q_{i}^{a_{i}^{*}}\left(x, a_{i}^{\prime}\right)$. Suppose $p_{i}^{a_{i}^{*}}\left(x, a_{i}^{\prime}\right)<q_{i}^{a_{i}^{*}}\left(x, a_{i}^{\prime}\right)$. Then by an earlier result, it follows that for every $\hat{\lambda}_{-i} \in \mathcal{M}_{-i}, \hat{\lambda}_{-i}\left(a_{-i}^{*}\right)=q_{i}^{a_{i}^{*}}\left(x, a_{i}^{\prime}\right)$ implies $\Delta \mathcal{U}_{i}\left(a_{i}^{*}, a_{i}^{\prime}, \hat{\lambda}_{-i}, x\right)>0$, contradicting the fact that $q_{i}^{a_{i}^{*}}\left(x, a_{i}^{\prime}\right)$ is achieved on $\psi(x)$.

Proposition 12. Let $\Gamma$ be a global game and $a^{*}$ a profile of actions.

For $i \in \mathcal{I}$ and $a_{i}^{\prime} \neq a_{i}^{*}$, suppose $\psi$ is nonempty-valued on $X^{\prime} \subset \mathbb{R}$. Then

1. $\psi$ is upper-hemicontinuous on $X^{\prime}$

2. The function $p_{i}^{a_{i}^{*}}\left(x, a_{i}^{\prime}\right)$ is upper-semicontinuous on $X^{\prime}$

Proof. To prove statement (1), suppose $\left(x_{n}\right)$ is a sequence in $X^{\prime}$ and $x_{n} \rightarrow x \in X^{\prime}$. Suppose $\lambda_{-i}^{n} \in \psi\left(x_{n}\right)$ and $\lambda_{-i}^{n} \rightarrow \lambda_{-i} \in \mathcal{M}_{-i}$. Then for every $n, \Delta \mathcal{U}_{i}\left(a_{i}^{*}, a_{i}^{\prime}, \lambda_{-i}^{n}, x_{n}\right)=0$, and by continuity of $\Delta \mathcal{U}_{i}$ in $\left(\lambda_{-i}, x\right)$, it follows that $\Delta \mathcal{U}_{i}\left(a_{i}^{*}, a_{i}^{\prime}, \lambda_{-i}, x_{n}\right)=0$, whence $\lambda_{-i} \in \psi(x)$. Statement (2) now follows from the theorem of the maximum.

We now complete the proof of Proposition 6.

Proposition 13. Let $\Gamma$ be a parameterized game and $a^{*}$ a profile of actions.

For $i \in \mathcal{I}$ and $X^{\prime} \subset \mathbb{R}$, if for every $x \in X^{\prime}$, $a_{i}^{*}$ is the unique best response of player $i$ to $a_{-i}^{*}$ at $x$, then

1. For every $a_{i}^{\prime} \neq a_{i}^{*}, p_{i}^{a_{i}^{*}}\left(x, a_{i}^{\prime}\right)$ is upper-semicontinuous on $X^{\prime}$, and

2. $p_{i}^{a_{i}^{*}}(x)$ is upper-semicontinuous on $X^{\prime}$.

Proof. To prove statement (1), we show that for every sequence $\left(x_{n}\right)_{n=1}^{\infty}$ in $X^{\prime}, x_{n} \rightarrow x \in$ $X^{\prime} \Rightarrow \lim \sup _{n} p_{i}^{a_{i}^{*}}\left(x_{n}, a_{i}^{\prime}\right) \leq p_{i}^{a_{i}^{*}}\left(x, a_{i}^{\prime}\right)$.

As case 1 , suppose $\exists N$ such that $\forall n \geq N, x_{n} \in D_{i}^{a_{i}^{*}}\left(a_{i}^{\prime}\right)$. Then $\forall n \geq N, p_{i}^{a_{i}^{*}}\left(x_{n}, a_{i}^{\prime}\right)=0$, whence $\lim \sup _{n} p_{i}^{a_{i}^{*}}\left(x_{n}, a_{i}^{\prime}\right)=0 \leq p_{i}^{a_{i}^{*}}\left(x, a_{i}^{\prime}\right)$.

As case 2, suppose $\forall N, \exists n \geq N, x_{n} \notin D_{i}^{a_{i}^{*}}\left(a_{i}^{\prime}\right)$. Let $\hat{\mathbb{N}}=\left\{n \in \mathbb{N} \mid x_{n} \notin D_{i}^{a_{i}^{*}}\left(a_{i}^{\prime}\right)\right\}$, and for each $n \in \mathbb{N}$, let $m(n)=\min \{j \in \hat{\mathbb{N}} \mid j \geq n\}$, and consider the sequence $y_{n}=$ $x_{m(n)}$. Notice that for all $n, y_{n} \notin D_{i}^{a_{i}^{*}}\left(a_{i}^{\prime}\right)$. Moreover, $n \in \hat{\mathbb{N}} \Rightarrow y_{n}=x_{n}$, and therefore, $p_{i}^{a_{i}^{*}}\left(y_{n}, a_{i}^{\prime}\right)=p_{i}^{a_{i}^{*}}\left(x_{n}, a_{i}^{\prime}\right)$, and $n \notin \hat{\mathbb{N}} \Rightarrow p_{i}^{a_{i}^{*}}\left(y_{n}, a_{i}^{\prime}\right) \geq 0=p_{i}^{a_{i}^{*}}\left(x_{n}, a_{i}^{\prime}\right)$. Consequently, $\lim \sup _{n} p_{i}^{a_{i}^{*}}\left(y_{n}, a_{i}^{\prime}\right) \geq \lim \sup _{n} p_{i}^{a_{i}^{*}}\left(x_{n}, a_{i}^{\prime}\right)$. Furthermore, $\left|y_{n}-x\right|=\left|x_{m(n)}-x\right| \rightarrow$ 0 , as $n \rightarrow \infty$, whence $y_{n} \rightarrow x$. Using case 1 , it follows that $\limsup _{n} p_{i}^{a_{i}^{*}}\left(x_{n}, a_{i}^{\prime}\right) \leq$ $\lim \sup _{n} p_{i}^{a_{i}^{*}}\left(y_{n}, a_{i}^{\prime}\right) \leq p_{i}^{a_{i}^{*}}\left(x, a_{i}^{\prime}\right)$. 
Statement (2) now follows because $p_{i}^{a_{i}^{*}}(x)=\max _{a_{i}^{\prime} \neq a_{i}^{*}} p_{i}^{a_{i}^{*}}\left(x, a_{i}^{\prime}\right)$ and the maximum of finitely many upper semicontinuous functions is upper semicontinuous. 